\title{
Systematic Review and Meta-Analysis of the Y-Balance Test Lower Quarter: Reliability, Discriminant Validity, and Predictive Validity
}

Phillip Plisky1, Katherine Schwartkopf-Phifer², Bethany Huebner ${ }^{1}$, Mary Beth Garner ${ }^{3}$, Garrett Bullock ${ }^{4}$ a

1 Physical Therapy, University of Evansville; ProRehab-PC, 2 Physical Therapy, University of Evansville; Rehabitlitation \& Performance Institute, 3 Physical Therapy, University of Evansville, ${ }^{4}$ Department of Orthopaedic Surgery, Wake Forest School of Medicine; Centre for Sport, Exercise and Osteoarthritis Research Versus Arthritis, University of Oxford

Keywords: y-balance test lower quarter, dynamic balance, single leg balance, star excursion balance test

https://doi.org/10.26603/001c.27634

\section{International Journal of Sports Physical Therapy}

Vol. 16, Issue 5, 2021

\section{Background}

Deficits in dynamic neuromuscular control have been associated with post-injury sequelae and increased injury risk. The Y-Balance Test Lower Quarter (YBT-LQ) has emerged as a tool to identify these deficits.

\section{Purpose}

To review the reliability of the YBT-LQ, determine if performance on the YBT-LQ varies among populations (i.e., sex, sport/activity, and competition level), and to determine the injury risk identification validity of the YBT-LQ based on asymmetry, individual reach direction performance, or composite score.

\section{Study Design}

Systematic Review

\section{Methods}

A comprehensive search was performed of 10 online databases from inception to October 30, 2019. Only studies that tested dynamic single leg balance using the YBT-LQ were included. Studies were excluded if the Y-Balance Test kit was not utilized during testing or if there was a major deviation from the Y-Balance test procedure. For methodological quality assessment, the modified Downs and Black scale and the Newcastle-Ottawa Scale were used.

\section{Results}

Fifty-seven studies (four in multiple categories) were included with nine studies assessing reliability, 36 assessing population differences, and 16 assessing injury prediction were included. Intra-rater reliability ranged from $0.85-0.91$. Sex differences were observed in the posteromedial direction (males: 109.6 [95\%CI 107.4-111.8]; females: 102.3 [95\%CI 97.2-107.4; $\mathrm{p}=0.01]$ ) and posterolateral direction (males: 107.0 [95\%CI 105.0-109.1]; females: 102.0 [95\%CI 97.8-106.2]). However, no difference was observed between sexes in the anterior reach direction (males: 71.9 [95\%CI 69.5-74.5]; females: 70.8 [95\% CI 65.7-75.9]; $\mathrm{p}=0.708$ ). Differences in composite score were noted between soccer (97.6; 95\%CI 95.9-99.3) and basketball (92.8; 95\%CI 90.4-95.3; $\mathrm{p}<0.01$ ), and baseball (97.4; 95\%CI 94.6-100.2) and basketball (92.8; 95\%CI 90.4-95.3; $\mathrm{p}=0.02)$. Given the heterogeneity of injury prediction studies, a meta-analysis of these data was not possible. Three of the 13 studies reported a relationship between anterior reach asymmetry reach and injury risk, three of 10 studies for posteromedial and posterolateral reach asymmetry,

\footnotetext{
a Corresponding author:

Garrett S. Bullock PT, DPT, DPhil

Department of Orthopaedic Surgery

Wake Forest School of Medicine

Winston-Salem, NC

gbullock@wakehealth.edu
} 
and one of 13 studies reported relationship with composite reach asymmetry.

\section{Conclusions}

There was moderate to high quality evidence demonstrating that the YBT-LQ is a reliable dynamic neuromuscular control test. Significant differences in sex and sport were observed. If general cut points (i.e., not population specific) are used, the YBT-LQ may not be predictive of injury. Clinical population specific requirements (e.g., age, sex, sport/ activity) should be considered when interpreting YBT-LQ performance, particularly when used to identify risk factors for injury.

\section{Level of Evidence}

$1 b$

\section{INTRODUCTION}

Despite increased evidence on injury prevention and identification, injuries ranging from minor to career-limiting continue to rise. ${ }^{1,2}$ Deficits in lower extremity dynamic neuromuscular control have been implicated as an injury risk factor and have been observed after lower extremity injury. ${ }^{3-6}$ Interventions to improve lower extremity dynamic neuromuscular control have been utilized as a component in multiple injury prevention programs. Specifically, researchers have observed that athletes who participated in an injury prevention program displayed improved lower extremity dynamic neuromuscular control. ${ }^{7,8}$ One study observed that the intervention group who was most compliant demonstrated the greatest lower extremity dynamic neuromuscular control improvement, and sustained lower extremity injuries at decreased rates. ${ }^{8}$ Additionally, health care practitioners frequently utilize dynamic neuromuscular control as an outcome measure for return to sport criterion. Thus, there is a need for a lower extremity dynamic neuromuscular control test that identifies athletes at increased injury risk, captures changes that may occur with intervention, and evaluates return to sport readiness (i.e., ensure motor control deficits that occur after injury have normalized). In order to be useful in a sports setting the test would need to be valid and easy to use.

The Star Excursion Balance Test (SEBT) and Y-Balance Test Lower Quarter (YBT-LQ) have been studied and used extensively for the determination of physical readiness and injury risk identification, return to sport testing, and prepost intervention measurement. ${ }^{6,9}$ The SEBT, through a systematic review, has been found to be reliable, valid, and responsive to specific dynamic neuromuscular control training for injured and healthy athletic populations. ${ }^{6}$ The advantage of the SEBT and YBT-LQ is that they test neuromuscular control at the limits of stability, which may allow for identification and magnification of subtle deficits and asymmetry. 6

The YBT-LQ was developed from the SEBT in order to improve the reliability and field expediency of the SEBT. ${ }^{9}$ The YBT-LQ was simplified to use only the most reliable three reach directions (compared to eight reach directions with the SEBT). While both tests require dynamic neuromuscular control at the limits of stability, there are differences between the tests. The YBT-LQ uses a standardized approach via a testing kit and revised protocol to improve the reliability and testing speed. Protocol revisions include: heel of stance foot is allowed to raise, no touch down is al- lowed with reaching limb, and kit incorporates a standard reach height off the ground is used. ${ }^{9}$

While the efficiency of the test may have been improved, these differences in test procedures can alter performance, leading researchers to conclude that the SEBT and YBT-LQ are not interchangeable. ${ }^{10,11}$ Coughlan et al. ${ }^{10}$ compared the performance on the SEBT and YBT-LQ, and found that healthy males reached farther on the SEBT in the anterior direction, but had similar reach distances in the posterior directions. ${ }^{10}$ Fullam et al. ${ }^{11}$ examined the kinematic differences between the SEBT and YBT-LQ. It was confirmed that healthy males reached farther in the anterior direction, and from a kinematic perspective, the YBT-LQ anterior reach had greater hip flexion. ${ }^{11}$ These differences may be due to procedural differences or the use of a standardized YBT-LQ test kit. In addition to the differences in results between the YBT-LQ and SEBT, researchers have found that there may be differences in performance based on sex, sport and competition level in both tests. ${ }^{3,4}$ Differences have been reported between subject performance on the YBT-LQ based on country of origin, 12 as well as, competition level. ${ }^{13,14}$ However, it is uncertain whether these findings are isolated to these populations or represent a true difference in performance among populations.

While a systematic review has been performed on the reliability and discriminant validity of the SEBT, the YBTLQ has not undergone a similar rigorous analysis regarding its effectiveness regarding injury risk identification. ${ }^{6}$ In the SEBT systematic review, the YBT-LQ was described as reliable, but only one study was available; thus, there is a need to investigate and summarize the YBT-LQ literature. ${ }^{6}$ The purpose of this systematic review and meta-analysis was to review the reliability of the YBT-LQ, determine if performance on the YBT-LQ varies among populations (i.e., sex, sport/activity, and competition level), and to determine the injury risk identification validity of the YBT-LQ based on asymmetry, individual reach direction performance, or composite score.

\section{METHODS}

\section{STUDY DESIGN}

A systematic review was performed on the reliability, validity, and population differences of the YBT-LQ. The Preferred Reporting Items for Systematic Reviews and Meta-Analyses (PRISMA) guidelines were utilized to conduct and report this review. 15 This review was prospectively registered with 
Prospero CRD42018090102.

\section{SEARCH STRATEGY}

A comprehensive computerized search was performed, employing online databases (MEDLINE, CINAHL, Cochrane, Embase, SPORTDiscus, Health Source-Consumer Edition, Health Source: Nursing/Academic Edition, SocINDEX, and Social Sciences), from inception to October 30, 2019. Medical subject headings (MeSH) and keywords were utilized for "dynamic balance," "Y-Balance Test," "Star Excursion Balance Test," and "single leg balance." The full search strategy entailed "y balance test" "[All Fields] OR "star excursion balance test*" [All Fields] OR YBT[All Fields] OR SEBT[All Fields]. References were tracked in Covidence systematic review software (Veritas Health Innovation, Melbourne, Australia).

\section{ELIGIBILITY CRITERIA}

Studies examining the YBT-LQ were included if they met the following criteria: 1) tested dynamic single leg balance using the YBT-LQ; 2) full-text articles were written in English. Study exclusion criteria consisted of 1) studies that did not use the Y-Balance Test kit during testing; 2) major deviation from the Y-Balance Test procedure (e.g., stance foot heel kept down); 3) the Y-Balance Test Upper Quarter procedure was utilized instead of the YBT-LQ; 4) conference abstracts or non-peer-reviewed papers.

\section{STUDY SELECTION}

Four reviewers (GB, MG, BH, KS) were split into pairs, and each pair independently assessed half of the selected studies. Title and abstracts were first screened using inclusion and exclusion criteria. Four reviewers independently, who were all physical therapists and specialized in sports medicine, executed full-text review following title and abstract screening. Any conflicts were first discussed within the four reviewers. If a consensus could not be reached, another reviewer $(\mathrm{PJ})$, who is a physical therapist, athletic trainer, $\mathrm{PhD}$, with over twenty years' experience in sports medicine, was utilized to determine final study eligibility. Following full-text review, a hand search was performed for any studies missed within the initial search.

\section{DATA EXTRACTION}

Data were extracted into a customized Excel spreadsheet (Version 2013, Microsoft, Redmond, Washington, United States) in three domains: reliability, population differences, and injury prediction. Two reviewers verified data for each domain. Disagreements concerning data domain placement were resolved by a third reviewer (PJ). Data elements included study characteristics (e.g., publication data, study design, and population), YBT-LQ methodology, and results (number of injuries, reach distance, reach asymmetry, and reliability).

\section{QUALITY ASSESSMENT}

All three domains (reliability, population differences, and injury prediction) were each analyzed by two independent reviewers (GB, MG, BH, KS). A third reviewer $(\mathrm{PJ})$ resolved any quality assessment disagreements. The Oxford Centre for Evidence-Based Medicine (OCEBM) levels of evidence (Level I to IV) ${ }^{16}$ was used to discern study design. The YBTLQ methodology was specifically assessed for uniformity. ${ }^{6}$ The YBT-LQ protocol factors that were assessed included the use of shoes during testing, the use of the average or maximum reach for each reach direction, hand placement during testing, number of practice trials, and number of data collection trials. ${ }^{6}$ The modified Downs and Black tool was utilized for methodological assessment for studies within the reliability and population differences domains. ${ }^{17,18}$ The modified Downs and Black tool has been shown to be reliable and valid. ${ }^{17}$ This methodological tool was scored on a scale of 0 to 15 . The scoring system has a stratified ranking, with a score of 12 or greater deemed high quality, a score of 10 to 11 deemed moderate quality, and a score at or below 9 deemed low quality. ${ }^{18}$ The NewcastleOttawa Scale (NOS) was utilized for methodological assessment for studies within the injury prediction domain. The NOS incorporates a 'star system' for three broad perspectives: the selection of the study groups (four questions); the comparability of the groups (one question); and the ascertainment of outcome of interest (three questions). Multiple questions can have more than one star, which may result in the number of stars totaling greater than total number of questions. ${ }^{19}$

\section{STATISTICAL ANALYSES}

Percentage agreement and Cohen Kappa statistics were calculated to provide absolute agreement between raters in SPSS 23 (SPSS Inc, IBM, Chicago, Illinois). The extracted data were aggregated into three domains: reliability, population differences, and injury prediction. Reliability data were summarized in a narrative fashion. The population differences domain data were analyzed by pooling the study means through a random effects inverse variance approach, originally described by DerSimonian and Laird. ${ }^{20}$ Studies that reported more than one individual cohort were each calculated as individual studies. Heterogeneity was assessed with the Cochrane $\mathrm{Q}$ and $\mathrm{I}^{2}$ with high heterogeneity designated by a Q p-value $<0.10$ and $\mathrm{I}^{2}>50 \%$. Meta-analysis was used to combine and summarize the data. In outcomes related meta-analysis, high heterogeneity indicates that there is large variation in study outcomes between studies and that results should not be pooled or combined. In this meta-analysis, high heterogeneity was observed indicating that there indeed may be differences in performance on the YBT-LQ among populations (i.e., age, sex, sport, activity, occupation, and injury status). Through an abundance of caution, a random effects model was used assuming that even within populations, results fall in a normal distribution. Data subdivisions were first grouped by sex for each YBT-LQ reach and composite score then analyzed through a series of $z$-tests $(p<0.05)$. Due to the differences found between sexes, and the paucity of female studies, only males were assessed for further subdivisions. Additionally, competition level was not able to be compared as there were no greater than two subgroups at each compe- 
tition level. Male sports differences (for all three YBT-LQ reaches and composite scores) were analyzed through oneway ANOVA with Tukey-Kramer $Q$ tests to localize pairwise differences based on pooled study means and variances $(\mathrm{p}<0.05) .{ }^{21}$ All meta-analyses were performed in $\mathrm{R}$ version 3.5.1 (R Core Team (2013). R: A language and environment for statistical computing (R Foundation for Statistical Computing, Vienna, Austria. URL http://www.R-project.org/), using the meta package. ${ }^{22}$ Given the heterogeneity in study design and data reporting, injury prediction data were summarized in a narrative fashion.

\section{RESULTS}

\section{STUDY SELECTION}

A total of 982 titles were identified through the initial database and hand searches. After removal of duplicate articles, 732 abstracts were reviewed for relevance. Substantial agreement was demonstrated in title and abstract screening $(\mathrm{k}=0.976, \mathrm{p}<0.01)$. Full text eligibility assessment of the remaining 411 articles resulted in 57 articles with 4 in multiple categories (Figure 1).3,9,12-14,23-50 Nine studies $9,25,33,40,46,51-54$ assessed reliability, 36 studies $^{12-14,23-30,32,35,37,38,41-45,47,49,54-67}$ examined differences in the performance on the YBT-LQ in different populations or reported mean performance on the YBTLQ in a specific population, and 16 studies $^{3,31,34,36,39,48,50,57,64,68-74}$ examined injury prediction (see Table 1). Substantial agreement was also observed for full text review $(\mathrm{k}=0.84, \mathrm{p}<0.01)$.

\section{QUALITY ASSESSMENT}

The NOS was used to assess quality of the included cohort studies $(\mathrm{n}=16)$. For the remaining 41 articles, the Downs and Black tool was used to assess quality. The scores of the included studies on the NOS ranged from 6-9 out of a possible 9, while the scores on the Downs and Black tool ranged from 7-13 out of a possible 15 (see summary in Table 1).

\section{RELIABILITY}

Nine studies $9,25,33,40,46,51-54$ assessed reliability of YBT-LQ (see Table 1). Intraclass correlation coefficients (ICCs) for intrarater reliability ranged from $0.57-0.82$ in adolescent populations, ${ }^{40}$ and $0.85-0.91$ in adult populations. ${ }^{9}$ Interrater reliability ICCs ranged from $0.81-1.00 .9,33,46,51$ Testretest reliability was assessed in five studies with ICCs ranging from $0.63-0.93 .25,33,52-54$

\section{SEX DIFFERENCES}

When sex was considered alone, differences were observed in the posteromedial direction (Male: 109.695\% CI 107.4-111.8; Female: 102.3 95\% CI 97.2-107.4; $\mathrm{p}<0.01$ ) and posterolateral direction (Male: 107.095\% CI 105.0-109.1; Female: $\quad 102.095 \% \quad$ CI $97.8-106.2$; $\mathrm{p}=0.036){ }^{12-14,25,27,37,43,44,58-61,63-66}$ However, no difference was observed between sexes in the anterior reach direction (Male: 71.995\% CI 69.5-74.5; Female: 70.895\% CI

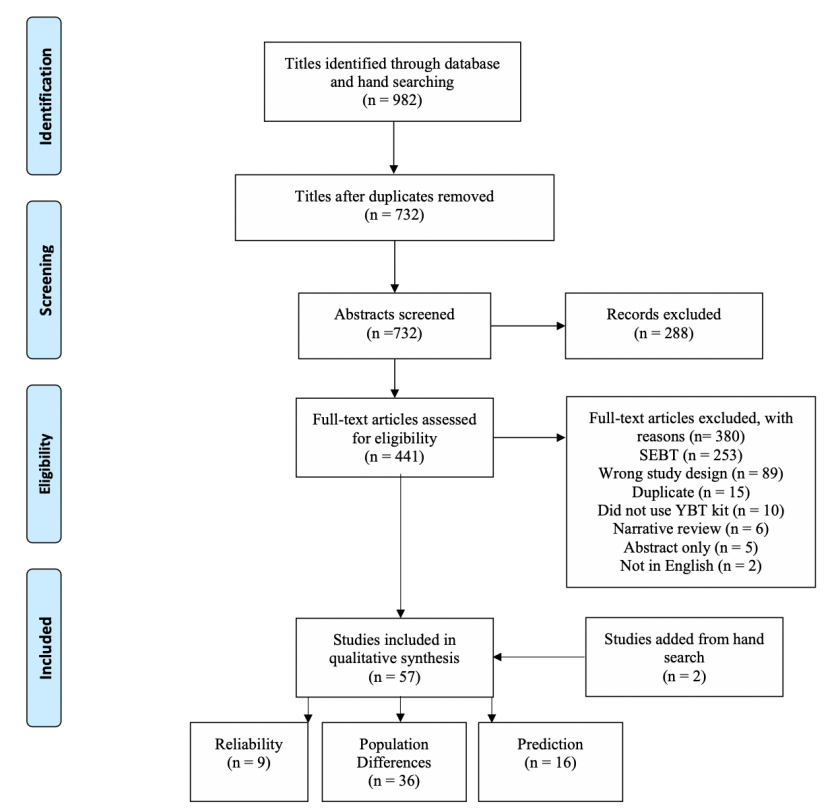

Figure 1. PRISMA study selection demonstrating the systematic review of the literature for reliability, validity, and population differences for the $\mathrm{Y}$ Balance Test Lower Quarter. PRISMA, Preferred Reporting Items for Systematic Reviews and MetaAnalyses.

65.7-75.9; $\mathrm{p}=0.708)^{12-14,25,27,37,44,54,57-61,63,65-67}$ or in composite score (Male: 95.895\% CI 94.5-97.2; Female: 95.395\% CI 92.9-97.8; $\mathrm{p}=0.75$ ) (Figure 2). ${ }^{12-14,24-30,32,37,38,42-44,55-57,59-66}$ However, there were significant differences based on sex, competition level, and sport throughout Figure 2. To illustrate, male Rwandan high school soccer players have a mean composite reach of 105.6 (95\% CI 102.99-108.21), ${ }^{12}$ while male professional basketball players have a mean composite reach of 92.0 (95\% CI 90.16-93.84). ${ }^{27}$ These scores also differ from female collegiate athletes, where a mean composite reach of 100.0 (95\% CI 98.87-101.13) was observed..$^{30}$

\section{COMPETITION LEVEL DIFFERENCES}

When competition level was considered alone (middle school, high school, college, professional), no differences were observed for the anterior $(p=0.05)$, posteromedial $(p=$ $0.69)$, posterolateral $(p=0.62)$, or composite score $(p=0.15)$ (Figure 3, $\underline{4}, \underline{5}, \underline{6}$ ). ${ }^{12-14,23-30,32,35,37,38,41-45,47,49,54-67}$

\section{SPORT DIFFERENCES}

In the anterior reach direction, a significant difference was observed between soccer and basketball athletes (Soccer: 76.095\% CI 73.6-78.4; Basketball: 70.595\% CI 67.7-73.2; p $<0.01) .{ }^{12-14,27}$ In the posteromedial reach direction, a significant difference was observed between soccer and basketball athletes (Soccer: 114.895\% CI 111.6-118.3; Basketball: 105.695\% CI 101.9-109.4; p < 0.01), and baseball and basketball athletes (Baseball: 113.895\% CI 109.5- 118.1; Basketball 105.695\% CI 101.9-109.4; $\mathrm{p}<0.01) .{ }^{12-14,27}$ In the 
Table 1: Study demographics, design, and risk of bias

\begin{tabular}{|c|c|c|c|c|c|c|}
\hline Author & $\begin{array}{l}\text { Level of } \\
\text { Evidence } \\
\text { (Study } \\
\text { Design) }\end{array}$ & $\begin{array}{l}\text { Study } \\
\text { Domain }\end{array}$ & Sport & $\begin{array}{c}\text { Competition } \\
\text { Level }\end{array}$ & $\begin{array}{c}\text { \# of } \\
\text { subjects } \\
\text { M:F }\end{array}$ & $\begin{array}{c}\text { Risk of Bias } \\
\text { (Downs and } \\
\text { Black) }\end{array}$ \\
\hline $\begin{array}{c}\text { Alnahdi et al. } \\
2014\end{array}$ & $\begin{array}{l}4 \text { (Case } \\
\text { series) }\end{array}$ & $\begin{array}{l}\text { Population } \\
\text { differences }\end{array}$ & - & - & $30: 31$ & $13 / 15$ \\
\hline $\begin{array}{l}\text { Avery et al. } \\
2017\end{array}$ & $\begin{array}{l}4 \text { (Case } \\
\text { series) }\end{array}$ & $\begin{array}{l}\text { Reliability } \\
\text { and } \\
\text { Population } \\
\text { differences }\end{array}$ & Ice Hockey & Youth & $36: 0$ & $12 / 15$ \\
\hline $\begin{array}{l}\text { Benis et al. } \\
\quad 2016\end{array}$ & $\begin{array}{c}1 \\
\text { (Randomized } \\
\text { controlled } \\
\text { trial) }\end{array}$ & $\begin{array}{l}\text { Reliability } \\
\text { and } \\
\text { Population } \\
\text { differences }\end{array}$ & Basketball & Elite & $0: 28$ & $13 / 15$ \\
\hline $\begin{array}{l}\text { Bonato et al. } \\
\quad 2017\end{array}$ & $\begin{array}{c}1 \\
\text { (Randomized } \\
\text { controlled } \\
\text { trial) }\end{array}$ & $\begin{array}{l}\text { Population } \\
\text { differences }\end{array}$ & Basketball & Elite & $0: 160$ & $13 / 15$ \\
\hline $\begin{array}{l}\text { Booysen et al. } \\
2015\end{array}$ & $\begin{array}{l}4 \text { (Case } \\
\text { series) }\end{array}$ & $\begin{array}{l}\text { Population } \\
\text { differences }\end{array}$ & Soccer & $\begin{array}{l}\text { University \& } \\
\text { Elite }\end{array}$ & $50: 0$ & $12 / 15$ \\
\hline $\begin{array}{l}\text { Bullock et al. } \\
\quad 2016\end{array}$ & $\begin{array}{l}4 \text { (Case } \\
\text { series) }\end{array}$ & $\begin{array}{l}\text { Population } \\
\text { differences }\end{array}$ & Basketball & $\begin{array}{c}\text { Middle } \\
\text { School High } \\
\text { School } \\
\text { Collegiate } \\
\text { Professional }\end{array}$ & $\begin{array}{c}88: 0 \\
105: 0 \\
46: 041: 0\end{array}$ & $12 / 15$ \\
\hline $\begin{array}{l}\text { Butler et al. } \\
\quad 2012\end{array}$ & $\begin{array}{l}4 \text { (Case } \\
\text { series) }\end{array}$ & $\begin{array}{l}\text { Population } \\
\text { differences }\end{array}$ & Soccer & $\begin{array}{l}\text { High School } \\
\text { Collegiate } \\
\text { Professional }\end{array}$ & $\begin{array}{c}38: 037: 0 \\
44: 0\end{array}$ & $12 / 15$ \\
\hline $\begin{array}{l}\text { Butler et al } \\
2013\end{array}$ & $\begin{array}{l}4 \text { (Case } \\
\text { series) }\end{array}$ & $\begin{array}{l}\text { Population } \\
\text { differences }\end{array}$ & Soccer & Adolescent & $26: 0$ & $12 / 15$ \\
\hline $\begin{array}{l}\text { Butler et al. } \\
\quad 2016\end{array}$ & $\begin{array}{l}4 \text { (Case } \\
\text { series) }\end{array}$ & $\begin{array}{l}\text { Population } \\
\text { differences }\end{array}$ & Baseball & $\begin{array}{l}\text { High School } \\
\text { Collegiate } \\
\text { Professional }\end{array}$ & $\begin{array}{c}\text { 88:0 78:0 } \\
90: 0\end{array}$ & $13 / 15$ \\
\hline $\begin{array}{l}\text { Chaouachi et } \\
\text { al. } 2017\end{array}$ & $\begin{array}{c}1 \\
\text { (Randomized } \\
\text { control trial) }\end{array}$ & $\begin{array}{l}\text { Population } \\
\text { differences }\end{array}$ & Soccer & $\begin{array}{l}\text { Adolescent } \\
\text { Elite }\end{array}$ & $26: 0$ & $12 / 15$ \\
\hline $\begin{array}{l}\text { Chimera et al. } \\
2015\end{array}$ & $\begin{array}{l}3 \text { (Case } \\
\text { control) }\end{array}$ & $\begin{array}{l}\text { Population } \\
\text { differences }\end{array}$ & $\begin{array}{c}\text { Basketball } \\
\text { Basketball Cheer } \\
\text { \& Dance Cross } \\
\text { Country Cross } \\
\text { Country Football } \\
\text { Golf Soccer } \\
\text { Swimming \& } \\
\text { Diving Tennis } \\
\text { Tennis Track \& } \\
\text { Field Track \& Field } \\
\text { Volleyball }\end{array}$ & $\begin{array}{l}\text { Division I } \\
\text { Collegiate }\end{array}$ & $\begin{array}{c}9: 00: 2 \\
0: 4 \text { 13:0 } \\
0: 1769: 0 \\
0: 30: 28 \\
0: 17 \text { 5:0 } \\
0: 5 \text { 7:0 } \\
0: 30: 8\end{array}$ & $12 / 15$ \\
\hline $\begin{array}{l}\text { Chimera et al. } \\
2016\end{array}$ & $\begin{array}{l}4 \text { (Case } \\
\text { series) }\end{array}$ & $\begin{array}{l}\text { Population } \\
\text { differences }\end{array}$ & Rowing & $\begin{array}{l}\text { Adolescent } \\
\text { Varsity } \\
\text { Adolescent } \\
\text { Novice }\end{array}$ & $0: 310: 21$ & $11 / 15$ \\
\hline $\begin{array}{l}\text { Engquist et al. } \\
2015\end{array}$ & $\begin{array}{l}4 \text { (Case } \\
\text { series) }\end{array}$ & $\begin{array}{l}\text { Population } \\
\text { differences }\end{array}$ & - & $\begin{array}{l}\text { Division I } \\
\text { Collegiate } \\
\text { General } \\
\text { College } \\
\text { students }\end{array}$ & $\begin{array}{l}88: 79 \\
31: 72\end{array}$ & $12 / 15$ \\
\hline $\begin{array}{l}\text { Faigenbaum et } \\
\text { al. } 2014\end{array}$ & $\begin{array}{c}2 \\
\text { (Randomized } \\
\text { control trial) }\end{array}$ & Reliability & - & - & $97: 91$ & $10 / 15$ \\
\hline $\begin{array}{l}\text { Gorman et al. } \\
2012\end{array}$ & $\begin{array}{l}3 \text { (Case } \\
\text { control) }\end{array}$ & $\begin{array}{l}\text { Population } \\
\text { differences }\end{array}$ & $\begin{array}{c}\text { Single Sport Multi- } \\
\text { Sport }\end{array}$ & High School & $68: 24$ & $12 / 15$ \\
\hline Greenberg et & 2 & Reliability & Athletes & Adolescent & $0: 21$ & $11 / 15$ \\
\hline
\end{tabular}




\begin{tabular}{|c|c|c|c|c|c|c|}
\hline al. 2019 & $\begin{array}{l}\text { (Prospective } \\
\text { cohort) }\end{array}$ & & & & & \\
\hline $\begin{array}{l}\text { Hoch et al. } \\
2017\end{array}$ & $\begin{array}{l}3 \text { (Case } \\
\text { control) }\end{array}$ & $\begin{array}{l}\text { Population } \\
\text { differences }\end{array}$ & Field Hockey & Collegiate & $0: 20$ & $11 / 15$ \\
\hline $\begin{array}{c}\text { Hudson et al. } \\
2017\end{array}$ & $\begin{array}{l}4 \text { (Case } \\
\text { series) }\end{array}$ & $\begin{array}{l}\text { Population } \\
\text { differences }\end{array}$ & Volleyball & Collegiate & $0: 90$ & $11 / 15$ \\
\hline $\begin{array}{c}\text { Johnston et al. } \\
2019\end{array}$ & $\begin{array}{c}2 \\
\text { (Prospective } \\
\text { cohort) }\end{array}$ & $\begin{array}{l}\text { Population } \\
\text { differences }\end{array}$ & Rugby & $\begin{array}{l}\text { Under } 20 \\
\text { Senior }\end{array}$ & $\begin{array}{c}50: 0 \\
211: 0\end{array}$ & $11 / 15$ \\
\hline $\begin{array}{l}\text { Kenny et al. } \\
2018\end{array}$ & $\begin{array}{c}2 \\
\text { (Prospective } \\
\text { cohort) }\end{array}$ & Reliability & Dance & $\begin{array}{c}\text { Pre- } \\
\text { professional }\end{array}$ & $3: 35$ & $9 / 15$ \\
\hline $\begin{array}{l}\text { Krysak et al. } \\
2019\end{array}$ & $\begin{array}{l}\text { 2b (Cross- } \\
\text { sectional } \\
\text { cohort) }\end{array}$ & $\begin{array}{l}\text { Population } \\
\text { differences }\end{array}$ & Golf & $\begin{array}{l}\text { Middle High } \\
\text { School } \\
\text { College } \\
\text { Professional }\end{array}$ & $\begin{array}{c}53: 0 \\
129: 0 \\
207: 0 \\
29: 0\end{array}$ & $11 / 15$ \\
\hline $\begin{array}{l}\text { Lacey et al. } \\
2019\end{array}$ & $\begin{array}{l}4 \text { (Observa- } \\
\text { tional } \\
\text { repeated } \\
\text { measures) }\end{array}$ & Reliability & $\begin{array}{l}\text { Gaelic Football, } \\
\text { Hurling, Camogie, } \\
\text { Soccer, Rugby }\end{array}$ & $\begin{array}{l}\text { Local sports } \\
\text { clubs }\end{array}$ & 11:8 & $10 / 15$ \\
\hline $\begin{array}{l}\text { Linek et al. } \\
\quad 2017\end{array}$ & $\begin{array}{c}2 \\
\text { (Randomized } \\
\text { control trial) }\end{array}$ & Reliability & Soccer & $\begin{array}{l}\text { Adolescent } \\
\text { semi- } \\
\text { professional }\end{array}$ & $38: 0$ & $9 / 15$ \\
\hline $\begin{array}{l}\text { Lisman et al. } \\
\quad 2018\end{array}$ & $\begin{array}{l}4 \text { (Cross- } \\
\text { sectional) }\end{array}$ & $\begin{array}{l}\text { Population } \\
\text { differences }\end{array}$ & Football & $\begin{array}{l}\text { Middle High } \\
\text { School }\end{array}$ & 2952 & $12 / 15$ \\
\hline $\begin{array}{l}\text { Miller et al. } \\
2017\end{array}$ & $\begin{array}{l}3 \text { (Case } \\
\text { control) }\end{array}$ & $\begin{array}{l}\text { Population } \\
\text { differences }\end{array}$ & - & High School & $117: 178$ & $12 / 15$ \\
\hline $\begin{array}{l}\text { de la Motte et } \\
\text { al. } 2016 \text { A }\end{array}$ & $\begin{array}{l}4 \text { (Case } \\
\text { series) }\end{array}$ & $\begin{array}{l}\text { Population } \\
\text { differences }\end{array}$ & US Marines & - & $356: 0$ & $13 / 15$ \\
\hline $\begin{array}{l}\text { de la Motte et } \\
\text { al. 2016 B }\end{array}$ & $\begin{array}{l}4 \text { (Case } \\
\text { series) }\end{array}$ & $\begin{array}{l}\text { Population } \\
\text { differences }\end{array}$ & $\begin{array}{l}\text { US Military } \\
\text { applicants }\end{array}$ & - & $837: 147$ & $10 / 15$ \\
\hline $\begin{array}{l}\text { Linek et al. } \\
\quad 2019\end{array}$ & $\begin{array}{c}2 \mathrm{~b} \\
\text { (Cross- } \\
\text { sectional) }\end{array}$ & $\begin{array}{l}\text { Population } \\
\text { differences }\end{array}$ & Soccer & $\begin{array}{c}\text { Elite } \\
\text { Adolescents }\end{array}$ & $43: 0$ & $12 / 15$ \\
\hline $\begin{array}{l}\text { Lopez- } \\
\text { Valenciano et } \\
\text { al. } 2019\end{array}$ & $\begin{array}{c}3 \\
\text { (Cross- } \\
\text { sectional) }\end{array}$ & $\begin{array}{l}\text { Population } \\
\text { differences }\end{array}$ & Soccer & Professional & $88: 79$ & $10 / 15$ \\
\hline $\begin{array}{l}\text { Muehlbauer et } \\
\text { al. } 2019\end{array}$ & $\begin{array}{l}4 \text { (Cross- } \\
\text { sectional) }\end{array}$ & $\begin{array}{l}\text { Population } \\
\text { differences }\end{array}$ & Soccer & Sub-Elite & $76: 0$ & $11 / 15$ \\
\hline $\begin{array}{c}\text { O'Malley et al. } \\
2016\end{array}$ & $\begin{array}{l}4 \text { (Case } \\
\text { series) }\end{array}$ & $\begin{array}{l}\text { Population } \\
\text { differences }\end{array}$ & Gaelic Football & Collegiate & 78:0 & $13 / 15$ \\
\hline $\begin{array}{l}\text { Plisky et al. } \\
2009\end{array}$ & $\begin{array}{c}2 \\
\text { (Randomized } \\
\text { control trial) }\end{array}$ & Reliability & Soccer & Collegiate & $15: 0$ & $7 / 15$ \\
\hline $\begin{array}{l}\text { Rossler et al. } \\
\quad 2015\end{array}$ & $\begin{array}{c}1 \\
\text { (Randomized } \\
\text { control trial) }\end{array}$ & $\begin{array}{l}\text { Population } \\
\text { differences }\end{array}$ & Soccer & $\begin{array}{l}\text { Elementary/ } \\
\text { Middle } \\
\text { School }\end{array}$ & 157 & $12 / 15$ \\
\hline Ryu et al. 2019 & $\begin{array}{c}3 \\
\text { (Case } \\
\text { control) }\end{array}$ & $\begin{array}{l}\text { Population } \\
\text { differences }\end{array}$ & Baseball & Professional & $42: 0$ & $10 / 15$ \\
\hline $\begin{array}{l}\text { Schafer et al. } \\
2013\end{array}$ & $\begin{array}{c}2 \\
\text { (Randomized } \\
\text { control trial) }\end{array}$ & Reliability & Service Members & - & $53: 11$ & $10 / 15$ \\
\hline $\begin{array}{l}\text { Schlingermann } \\
\text { et al. } 2017\end{array}$ & $\begin{array}{c}1 \\
\text { (Randomized } \\
\text { control trial) }\end{array}$ & $\begin{array}{l}\text { Population } \\
\text { differences }\end{array}$ & Gaelic Football & Collegiate & $131: 0$ & $11 / 15$ \\
\hline $\begin{array}{l}\text { Slater et al. } \\
2018\end{array}$ & $\begin{array}{c}4 \\
\text { (Descriptive) }\end{array}$ & $\begin{array}{l}\text { Population } \\
\text { differences }\end{array}$ & Ice Skating & Senior Level & $17: 15$ & $10 / 15$ \\
\hline $\begin{array}{l}\text { Smith (Laura) } \\
\text { et al. } 2018\end{array}$ & $\begin{array}{l}2 \mathrm{~b} \text { (Cross- } \\
\text { sectional) }\end{array}$ & Reliability & $\begin{array}{l}\text { Football } \\
\text { Basketball }\end{array}$ & High School & $\begin{array}{c}30: 0 \\
12: 348: 0\end{array}$ & $12 / 15$ \\
\hline
\end{tabular}




\begin{tabular}{|c|c|c|c|c|c|c|}
\hline & & & $\begin{array}{l}\text { Lacrosse Softball } \\
\text { Soccer }\end{array}$ & & $0: 101: 15$ & \\
\hline $\begin{array}{l}\text { Smith (Joseph) } \\
\text { et al. } 2018\end{array}$ & $\begin{array}{l}2 b \text { (Cross- } \\
\text { sectional) }\end{array}$ & $\begin{array}{l}\text { Population } \\
\text { differences }\end{array}$ & Basketball Soccer & High School & $94: 91$ & $13 / 15$ \\
\hline $\begin{array}{c}\text { Teyhen et al. } \\
2014\end{array}$ & $\begin{array}{l}4 \text { (Case } \\
\text { series) }\end{array}$ & $\begin{array}{l}\text { Population } \\
\text { differences }\end{array}$ & Military & Army & $53: 11$ & $12 / 15$ \\
\hline $\begin{array}{l}\text { Teyhen et al. } \\
2016\end{array}$ & $\begin{array}{l}3 \text { (Case } \\
\text { control) }\end{array}$ & $\begin{array}{l}\text { Population } \\
\text { differences }\end{array}$ & Military & Army & 1380:86 & $13 / 15$ \\
\hline Author & $\begin{array}{l}\text { Level of } \\
\text { Evidence } \\
\text { (Study } \\
\text { Design) }\end{array}$ & $\begin{array}{l}\text { Study } \\
\text { Domain }\end{array}$ & Sport & $\begin{array}{l}\text { Competition } \\
\text { Level }\end{array}$ & $\begin{array}{c}\text { \# of } \\
\text { subjects } \\
\text { M:F }\end{array}$ & $\begin{array}{l}\text { Risk of Bias } \\
\text { (Newcastle) }\end{array}$ \\
\hline $\begin{array}{l}\text { Brumitt et al. } \\
2018\end{array}$ & $\begin{array}{c}2 \\
\begin{array}{c}\text { (Prospective } \\
\text { cohort) }\end{array}\end{array}$ & Predictive & Basketball & Collegiate & $169: 0$ & 8 \\
\hline $\begin{array}{l}\text { Butler et al. } \\
\quad 2013\end{array}$ & $\begin{array}{c}2 \\
\begin{array}{c}\text { (Prospective } \\
\text { cohort) }\end{array}\end{array}$ & Predictive & Football & Collegiate & $59: 0$ & 9 \\
\hline $\begin{array}{l}\text { Cosio-Lima et } \\
\text { al. } 2016\end{array}$ & $\begin{array}{c}2 \\
\text { (Prospective } \\
\text { cohort) }\end{array}$ & Predictive & Military & $\begin{array}{l}\text { Coast Guard } \\
\text { Maritime } \\
\text { Security } \\
\text { Response } \\
\text { Team } \\
\text { Candidates }\end{array}$ & $31: 0$ & 7 \\
\hline $\begin{array}{l}\text { de la Motte et } \\
\text { al. } 2019\end{array}$ & $\begin{array}{c}2 \\
\begin{array}{c}\text { (Prospective } \\
\text { cohort) }\end{array}\end{array}$ & Predictive & Military & - & 1433:281 & 10 \\
\hline $\begin{array}{l}\text { Gonell et al. } \\
\quad 2015\end{array}$ & $\begin{array}{c}2 \\
\begin{array}{c}\text { (Prospective } \\
\text { cohort) }\end{array}\end{array}$ & Predictive & Soccer & $\begin{array}{l}\text { Professional } \\
\text { Amateur }\end{array}$ & $34: 040: 0$ & 6 \\
\hline $\begin{array}{c}\text { Gonzalez et al. } \\
2018\end{array}$ & $\begin{array}{c}2 \\
\begin{array}{c}\text { (Prospective } \\
\text { cohort) }\end{array}\end{array}$ & Predictive & Rowing & $\begin{array}{l}\text { Division I } \\
\text { Collegiate }\end{array}$ & $0: 31$ & 11 \\
\hline $\begin{array}{l}\text { Hartley et al. } \\
\quad 2017\end{array}$ & $\begin{array}{c}2 \\
\text { (Prospective } \\
\text { cohort) }\end{array}$ & Predictive & $\begin{array}{c}\text { Baseball } \\
\text { Basketball } \\
\text { Football Lacrosse } \\
\text { Soccer Softball } \\
\text { Tennis Volleyball } \\
\text { Other }\end{array}$ & $\begin{array}{l}\text { Division II/ } \\
\text { NAIA } \\
\text { Collegiate }\end{array}$ & $\begin{array}{c}54: 0 \\
67: 35 \\
161: 0 \\
19: 0 \\
62: 48 \\
0: 3010: 0 \\
0: 30 \\
11: 24 \\
\end{array}$ & 6 \\
\hline $\begin{array}{l}\text { Johnston et al. } \\
2019\end{array}$ & $\begin{array}{c}2 \\
\text { (Prospective } \\
\text { cohort) }\end{array}$ & Predictive & Rugby & Elite & 109:0 & 9 \\
\hline Lai et al. 2017 & $\begin{array}{l}3 \text { (Case } \\
\text { control) }\end{array}$ & Predictive & - & $\begin{array}{l}\text { Division I } \\
\text { Collegiate } \\
\end{array}$ & $177: 117$ & 6 \\
\hline $\begin{array}{l}\text { Lisman et al. } \\
\quad 2019\end{array}$ & $\begin{array}{c}2 \\
\begin{array}{c}\text { (Prospective } \\
\text { cohort) }\end{array} \\
\end{array}$ & Predictive & $\begin{array}{c}\text { Football, Lacrosse, } \\
\text { Baseball }\end{array}$ & High School & $156: 0$ & 10 \\
\hline Ruffe et al & $\begin{array}{c}2 \\
\text { (Prospective } \\
\text { Cohort) }\end{array}$ & Predictive & Cross Country & High School & $68: 80$ & 10 \\
\hline $\begin{array}{l}\text { Siupsinskas et } \\
\text { al. } 2019\end{array}$ & $\begin{array}{c}2 \\
\begin{array}{c}\text { (Prospective } \\
\text { cohort) }\end{array}\end{array}$ & Predictive & Basketball & Professional & $0: 169$ & 10 \\
\hline $\begin{array}{l}\text { Smith et al. } \\
\quad 2015\end{array}$ & $\begin{array}{c}2 \\
\text { (Prospective } \\
\text { cohort) }\end{array}$ & Predictive & $\begin{array}{l}\text { Basketball Cross } \\
\text { Country Track \& } \\
\text { Field Tennis } \\
\text { Football Golf } \\
\text { Volleyball Soccer } \\
\text { Swimming \& } \\
\text { Diving }\end{array}$ & $\begin{array}{l}\text { Division I } \\
\text { Collegiate }\end{array}$ & $\begin{array}{c}9: 213: 17 \\
7: 35: 5 \\
68: 00: 3 \\
0: 80: 27 \\
0: 17\end{array}$ & 8 \\
\hline
\end{tabular}




\begin{tabular}{|c|c|c|c|c|c|c|}
\hline $\begin{array}{c}\text { Teyhen et al. } \\
2015\end{array}$ & $\begin{array}{c}2 \\
\text { (Prospective } \\
\text { prognostic) }\end{array}$ & Predictive & Military & $\begin{array}{c}\text { Army } \\
\text { Rangers }\end{array}$ & $188: 0$ & 9 \\
\hline $\begin{array}{c}\text { Vaulerin et al. } \\
2019\end{array}$ & $\begin{array}{c}2 \\
\text { (Prospective } \\
\text { cohort) }\end{array}$ & Predictive & Fire-fighters & - & $39: 0$ & 10 \\
\hline $\begin{array}{c}2 \\
\text { Wright et al. } \\
2017\end{array}$ & $\begin{array}{c}\text { (Prospective } \\
\text { cohort) }\end{array}$ & Predictive & $\begin{array}{c}\text { Volleyball Cross } \\
\text { Country Track \& } \\
\text { Field Lacrosse } \\
\text { Soccer }\end{array}$ & $\begin{array}{c}\text { Division I } \\
\text { Collegiate }\end{array}$ & $\begin{array}{c}144743 \\
34: 480: 3\end{array}$ & 6 \\
\hline
\end{tabular}

*A higher score on the Downs and Black and the Newcastle-Ottawa Scale indicates lower risk of bias

posterolateral reach direction, a significant difference was observed between soccer and basketball athletes(Soccer: 111.8, 95\%CI 108.5-115.0; Basketball: 102.095\% CI 101.3-104.4; $<$ 0.01), and baseball and basketball athletes (Baseball: 107.7 95\% CI 105.7-106.1; Basketball: 102.095\% CI 101.3-104.4; $\mathrm{p}<0.01) .{ }^{12-14,27}$ For composite score, there was a significant difference between soccer and basketball athletes (Soccer: 97.695\% CI 95.9-99.3; Basketball: $92.895 \%$ CI 90.4-95.3; p < 0.01) and baseball and basketball athletes (Baseball: 97.495\% CI 94.6-100.2; Basketball: 92.895\% CI 90.4-95.3; $\mathrm{p}=0.02) .{ }^{12-14,27}$

\section{INJURY PREDICTION}

A total of 16 studies $3,31,34,36,39,48,50,57,64,68-74$ investigated the association between YBT-LQ performance and injury risk: 12 investigated anterior reach asymmetry, 10 investigated asymmetries in the posteromedial and posterolateral directions, five studied individual reach directions, and 13 utilized composite scores. Populations studied include collegiate athletes $3,36,39,50,57,68,70(n=1,493)$, elite female basketball players ${ }^{73}(\mathrm{n}=169)$, male high school athletes ${ }^{72}$ $(\mathrm{n}=156)$, professional and amateur soccer athletes ${ }^{34}(\mathrm{n}=74)$, rugby players ${ }^{71}(\mathrm{n}=109)$, high school cross country runners $^{64}(\mathrm{n}=148)$, military personnel ${ }^{31,48,69}(\mathrm{n}=1919)$, and firefighters $^{74}(\mathrm{n}=39)$.

\section{ANTERIOR REACH ASYMMETRY}

Twelve studies $34,36,39,48,50,57,64,68,69,72-74$ investigated the injury prediction ability of the YBT-LQ anterior reach asymmetry (Subjects: $n=3,986$ ). Five of these studies $34,50,57,64,68$ examined anterior reach asymmetry using a cut off of $\geqslant 4$ $\mathrm{cm}$; three ${ }^{34,57,64}$ reported raw numbers of subjects falling above and below this cut off score. Due to the high level of methodological and reporting discrepancies in the available data, a meta-analysis was not able to be completed.

Smith et al. ${ }^{68}$ utilized the $4 \mathrm{~cm}$ threshold and found a relationship with future injury risk, reporting an OR of 2.20 (95\% CI 1.09-4.46). The remaining seven studies varied in interpretation of anterior reach performance. Five studies $^{39,48,69,72,74}$ utilized anterior asymmetry cut off values varying from $2-3 \mathrm{~cm}$; of these, Valuerin et al. ${ }^{74}$ found an asymmetry of $\geqslant 2 \mathrm{~cm}$ was predictive of ankle sprains. Siupsinksaks et al. ${ }^{73}$ reported only limb difference scores and did not find an association to injury in elite female basketball players. Hartley et al. ${ }^{36}$ created a reach distance cut off of $54.5 \%$ LL for the anterior reach and found a significant difference between injured and uninjured collegiate athletes. Populations and definition of injury and asymmetry varied between studies, however, the three studies identifying a relationship between injury risk and anterior reach all included collegiate or professional athletes.

POSTEROMEDIAL AND POSTEROLATERAL ASYMMETRY

Ten studies $3,34,36,39,57,64,68,72-74$ examined the relationship between posteromedial and/or posterolateral reach asymmetry and future injury risk. Gonell et al. ${ }^{34}$ reported an OR of 3.86 (95\%CI 1.46-10.95) for male soccer players with a posteromedial asymmetry of $4 \mathrm{~cm}$ or greater. No relationship was observed with posterolateral asymmetry. Four studies $57,64,68,72$ used the same $4 \mathrm{~cm}$ or greater asymmetry threshold for both the posteromedial and posterolateral directions, and found no relationship to future non-contact injuries in collegiate basketball players, high school cross country runners, collegiate athletes, or musculoskeletal injuries in male high school athletes, respectively. Hartley et al. ${ }^{36}$ also reported a significant difference in posteromedial reach asymmetry, with injured female athletes having a significantly reduced asymmetry compared to uninjured counterparts. Lai et al. ${ }^{39}$ reported asymmetries of $9 \mathrm{~cm}$ in the posteromedial reach direction and $3 \mathrm{~cm}$ in the posterolateral direction resulted in a sensitivity of $17.1 \%$ and $54.9 \%$ (respectively), while specificity was reported as $89.9 \%$ and $54.6 \%$ (respectively). Valuerin et al. ${ }^{74}$ and Siupsinskas et al. ${ }^{73}$ reported varying values for asymmetry in reach directions or limb differences, though no relationships to future injury risk were noted. Finally, Butler et al. ${ }^{3}$ did not observe significant differences in reach asymmetry between injured and uninjured football players.

\section{INDIVIDUAL REACH DIRECTIONS DISTANCE}

Five studies $34,36,50,69,71$ described the relationship between injury and individual reach directions. Four of these studies $^{34,36,50,69}$ reported normalized reach distances for all reach directions, with no significant difference noted between injured and uninjured subjects.

Johnston et al. ${ }^{71}$ examined the relationship between the anterior reach and future concussions. Using an inertial sensor, rugby players with increased sample entropy when reaching in the anterior direction were found to be 3 times more likely to sustain a concussion. No association between posteromedial and posterolateral reaches to concussion 


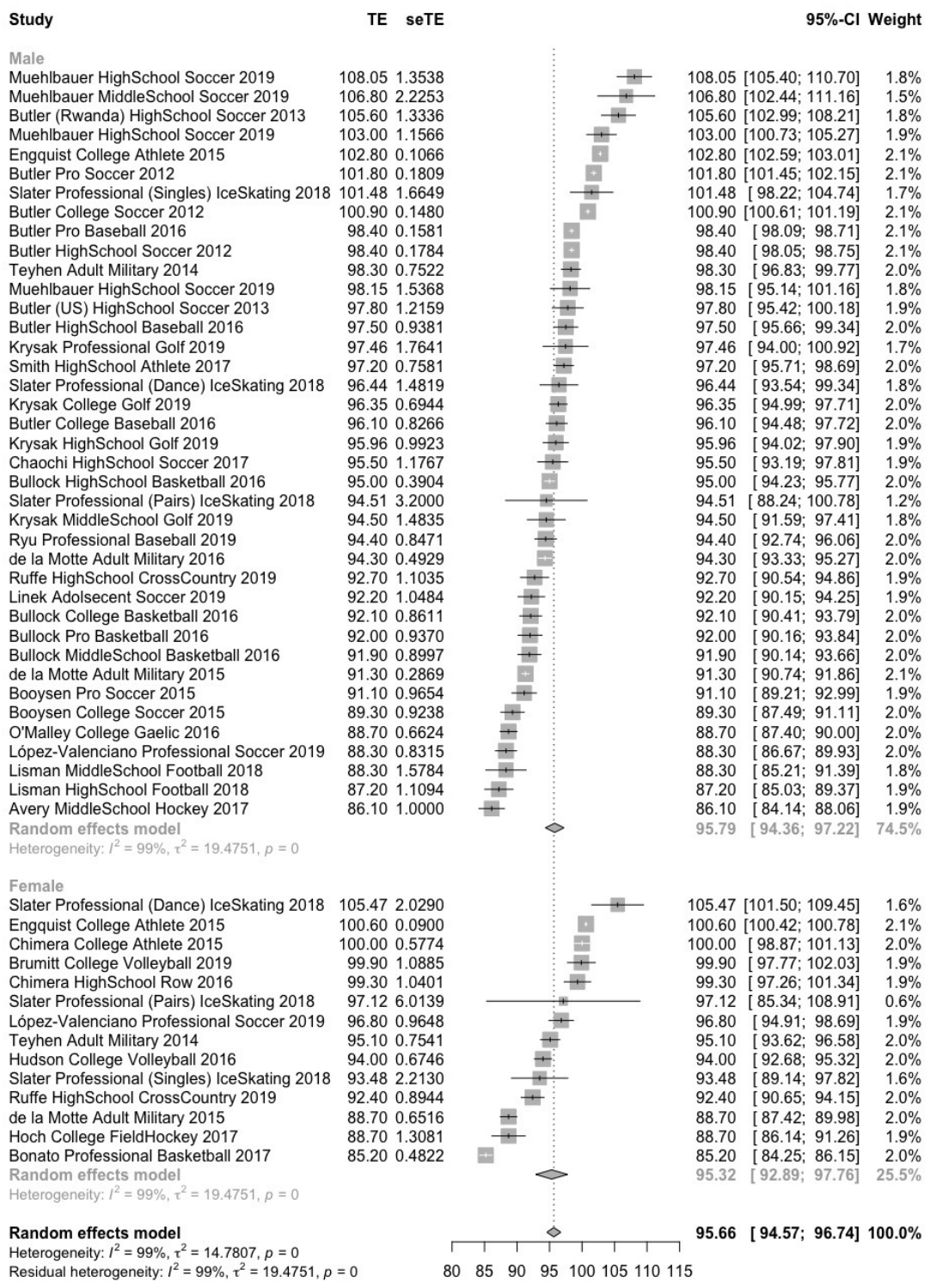

Figure 2: Pooled Y-Balance Test Composite Score, Grouped by Sex. MS = Middle School, HS = High School, Col $=$ College, Pro $=$ Professional, ADU $=$ Adult

was noted.

\section{COMPOSITE}

One 3 of 13 studies $3,31,34,39,48,50,57,64,68,69,72-74$ found a relationship between composite score and future injury. Butler et al. ${ }^{3}$ reported an odds ratio of 3.5 (95\%CI 2.4-5.3) when using a cutoff of $89.6 \%$ ( $\mathrm{SN}=100 \%, \mathrm{SP}=71.7 \%)$ in football players. Wright et al. ${ }^{50}$ and Brumitt et al. ${ }^{57}$ utilized different composite cutoffs for athletic teams, ranging from $89-94 \%$, all yielding non-significant likelihood ratios (ranges 0.55-1.32 and 0.50-1.70, respectively). Nine studies $^{31,34,39,48,68,69,72-74}$ did not report significant relation- ships between composite scores and future injury.

Three studies $34,64,69$ examined the relationship between composite score asymmetry and future injury. Gonell et al. ${ }^{34}$ and Ruffe et al. ${ }^{64}$ both utilized $12 \mathrm{~cm}$ or greater threshold for asymmetry and no relationship to injury was noted. De la Motte et al. ${ }^{69}$ found no significant differences in composite asymmetry between injured and uninjured military personnel $(\mathrm{p}=0.50)$.

\section{DISCUSSION}

Testing is an important function for researchers, health care providers, and performance professionals. Many decisions 


\begin{tabular}{|c|c|c|c|c|c|c|c|c|}
\hline Study & & seTE & & & & & $95 \%-\mathrm{Cl}$ & Weight \\
\hline Male & & & & & & & & \\
\hline Krysak HighSchool 2019 & 106.53 & 1.1507 & & & & $>106.53$ & {$[104.27 ; 108.79]$} & $2.3 \%$ \\
\hline Smith HighSchool 2018 & 92.40 & 0.8910 & & & + & 92.40 & [ $90.65 ; 94.15]$ & $2.4 \%$ \\
\hline Butler (Rwanda) HighSchool 2013 & 83.90 & 1.2159 & & + & & 83.90 & {$[81.52 ; 86.28]$} & $2.3 \%$ \\
\hline Muehlbauer HighSchool 2019 & 81.45 & 1.8548 & & + & & 81.45 & [ $77.81 ; 85.09]$ & $2.2 \%$ \\
\hline Muehlbauer MiddleSchool 2019 & 79.80 & 2.8218 & & $\stackrel{1}{1}$ & & 79.80 & {$[74.27 ; 85.33]$} & $2.0 \%$ \\
\hline Muehlbauer HighSchool 2019 & 77.20 & 1.2875 & & + & & 77.20 & {$[74.68 ; 79.72]$} & $2.3 \%$ \\
\hline Butler (US) HighSchool 2013 & 76.50 & 1.2944 & & + & & 76.50 & {$[73.96 ; 79.04]$} & $2.3 \%$ \\
\hline Butler HighSchool 2012 & 76.20 & 0.1622 & & + & & 76.20 & {$[75.88 ; 76.52]$} & $2.4 \%$ \\
\hline Bullock HighSchool 2016 & 76.00 & 0.5855 & & + & & 76.00 & {$[74.85 ; 77.15]$} & $2.4 \%$ \\
\hline Butler HighSchool 2016 & 75.90 & 0.8102 & & + & & 75.90 & {$[74.31 ; 77.49]$} & $2.4 \%$ \\
\hline Engquist College 2015 & 75.80 & 0.0959 & & + & & 75.80 & {$[75.61 ; 75.99]$} & $2.4 \%$ \\
\hline Bullock MiddleSchool 2016 & 73.70 & 0.8400 & & + & & 73.70 & {$[72.05 ; 75.35]$} & $2.4 \%$ \\
\hline Butler Professional 2016 & 73.20 & 0.6535 & & & & 73.20 & {$[71.92 ; 74.48]$} & $2.4 \%$ \\
\hline Muehlbauer HighSchool 2019 & 73.05 & 1.9777 & & & & 73.05 & [ $69.17 ; 76.93]$ & $2.2 \%$ \\
\hline Butler College 2012 & 72.80 & 0.1644 & & & & 72.80 & [ $72.48 ; 73.12]$ & $2.4 \%$ \\
\hline Krysak MiddleSchool 2019 & 72.27 & 1.1332 & & & & 72.27 & {$[70.05 ; 74.49]$} & $2.3 \%$ \\
\hline Butler Professional 2012 & 72.20 & 0.1960 & & & & 72.20 & {$[71.82 ; 72.58]$} & $2.4 \%$ \\
\hline Krysak College 2019 & 71.81 & 0.6436 & & & & 71.81 & {$[70.55 ; 73.07]$} & $2.4 \%$ \\
\hline Butler College 2016 & 71.60 & 0.7586 & & & & 71.60 & [ 70.11; 73.09] & $2.4 \%$ \\
\hline Slater (Singles) Professional 2018 & 69.49 & 1.6271 & $+\vdots$ & & & 69.49 & {$[66.30 ; 72.68]$} & $2.3 \%$ \\
\hline Krysak Professional 2019 & 69.33 & 2.0037 & + & & & 69.33 & {$[65.40 ; 73.26]$} & $2.2 \%$ \\
\hline de la Motte Adult 2016 & 68.20 & 0.3816 & + & & & 68.20 & {$[67.45 ; 68.95]$} & $2.4 \%$ \\
\hline Bullock College 2016 & 68.00 & 0.6738 & + & & & 68.00 & {$[66.68 ; 69.32]$} & $2.4 \%$ \\
\hline Linek HighSchool 2019 & 66.75 & 1.2664 & + & & & 66.75 & {$[64.27 ; 69.23]$} & $2.3 \%$ \\
\hline Slater (Dance) Professional 2018 & 66.56 & 2.0025 & $\div$ & & & 66.56 & {$[62.64 ; 70.49]$} & $2.2 \%$ \\
\hline O'Malley College 2016 & 65.80 & 0.8719 & + & & & 65.80 & {$[64.09 ; 67.51]$} & $2.4 \%$ \\
\hline Slater (Pairs) Professional 2018 & 65.45 & 2.3275 & + & & & 65.45 & {$[60.89 ; 70.01]$} & $2.1 \%$ \\
\hline Lisman MiddleSchool 2018 & 64.10 & 1.0399 & + & & & 64.10 & {$[62.06 ; 66.14]$} & $2.3 \%$ \\
\hline Ruffe HighSchool 2019 & 63.85 & 0.9277 & $\mp$ & & & 63.85 & {$[62.03 ; 65.67]$} & $2.4 \%$ \\
\hline Lisman HighSchool 2018 & 63.40 & 0.8043 & + & & & 63.40 & {$[61.82 ; 64.98]$} & $2.4 \%$ \\
\hline Ryu Professional 2019 & 63.15 & 0.9783 & + & & & 63.15 & {$[61.23 ; 65.07]$} & $2.4 \%$ \\
\hline Bullock Professional 2016 & 63.00 & 1.3275 & + & & & 63.00 & {$[60.40 ; 65.60]$} & $2.3 \%$ \\
\hline Miller HighSchool 2017 & 63.00 & 0.0925 & & & & 63.00 & {$[62.82 ; 63.18]$} & $2.4 \%$ \\
\hline Johnston HighSchool 2019 & 58.90 & 0.6647 & + & & & 58.90 & {$[57.60 ; 60.20]$} & $2.4 \%$ \\
\hline Johnston Professional 2019 & 57.20 & 0.3855 & + & & & 57.20 & {$[56.44 ; 57.96]$} & $2.4 \%$ \\
\hline Random effects model & & & & & & 71.93 & {$[69.50 ; 74.35]$} & $81.5 \%$ \\
\hline Heterogeneity: $I^{2}=100 \%, p=0$ & & & & & & & & \\
\hline & & & & & & & & \\
\hline Smith HighSchool 2018 & 95.00 & 0.9592 & & & 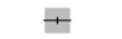 & 95.00 & [93.12;96.88] & $2.4 \%$ \\
\hline Slater (Dance) Professional 2018 & 75.46 & 1.8392 & & & & 75.46 & {$[71.86 ; 79.06]$} & $2.2 \%$ \\
\hline Engquist College 2015 & 73.40 & 0.0788 & & & & 73.40 & {$[73.25 ; 73.55]$} & $2.4 \%$ \\
\hline Slater (Pairs) Professional 2018 & 67.68 & 2.1249 & +1 & & & 67.68 & {$[63.52 ; 71.84]$} & $2.2 \%$ \\
\hline Brumitt College 2019 & 66.10 & 0.5615 & + & & & 66.10 & {$[65.00 ; 67.20]$} & $2.4 \%$ \\
\hline Slater (Singles) Professional 2018 & 66.06 & 1.7330 & 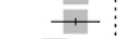 & & & 66.06 & {$[62.67 ; 69.46]$} & $2.2 \%$ \\
\hline Hoch College 2017 & 63.10 & 0.9481 & + & & & 63.10 & {$[61.24 ; 64.96]$} & $2.4 \%$ \\
\hline Ruffe HighSchool 2019 & 59.90 & 0.7211 & $=$ & & & 59.90 & {$[58.49 ; 61.31]$} & $2.4 \%$ \\
\hline Random effects model & & & & & & 70.85 & {$[65.76 ; 75.93]$} & $18.5 \%$ \\
\hline Heterogeneity: $I^{2}=99 \%, p<0.01$ & & & & & & & & \\
\hline Randon & & & & & & 71.72 & {$[69.83 ; 73.61]$} & $100.0 \%$ \\
\hline Heterogeneity: $I^{2}=100 \%, p=0$ & & & & 1 & 1 & & & \\
\hline Residual heterogeneity: $I^{2}=100 \%$, & & & 60 & 80 & 100 & & & \\
\hline
\end{tabular}

Figure 3: Pooled Y-Balance Test, Anterior Reach, Grouped by Sex. MS = Middle School, HS = High School, Col = College, Pro $=$ Professional, ADU $=$ Adult

hinge on test results, and it is essential to have validated tests in this process. While commonly used, the YBT-LQ has not been rigorously studied via systematic review and metaanalysis. This systematic review observed that the YBT-LQ is a highly reliable test. Dynamic balance differences were observed between sex, sport, and competition level, and asymmetry in the anterior reach demonstrated increased risk of lower extremity injury.

\section{RELIABILITY}

The YBT-LQ demonstrated high reliability over time and between raters. The high YBT-LQ reliability is comparable to the SEBT, which highlights the ability of the YBT-LO to accurately measure dynamic neuromuscular control. ${ }^{9}$ Higher variability in single session performance on the YBT-LQ in children may be due to the greater variability of balance performance seen in children. ${ }^{75}$

\section{DIFFERENCE IN YBT-LQ BY SEX, SPORT, AND COMPETITION LEVEL}

\section{SEX DIFFERENCES}

When sex was considered alone, differences were observed in the posteromedial and posterolateral directions, but no 


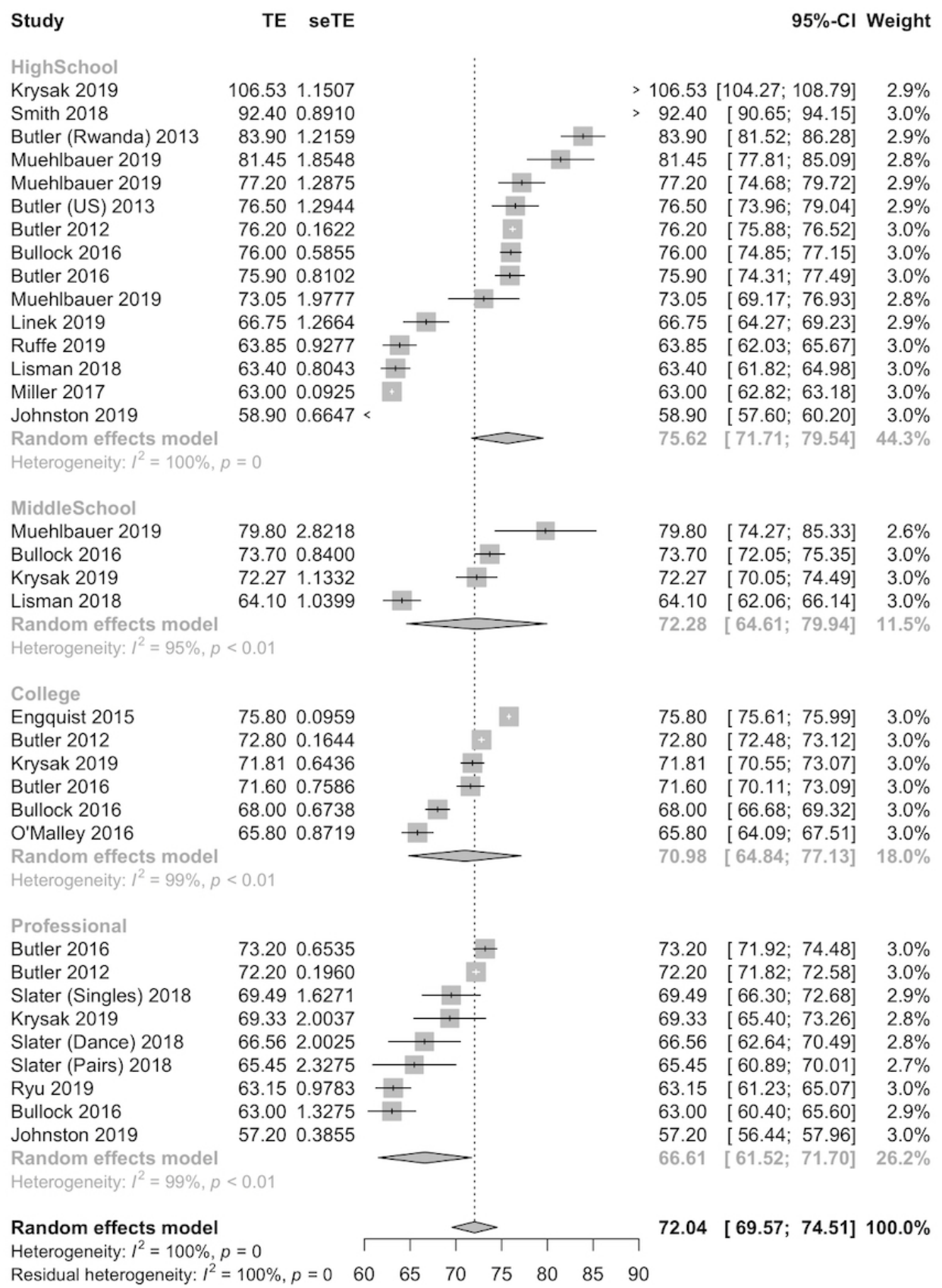

Figure 4: Pooled Y-Balance Test, Anterior Reach, Compared by Sport. MS = Middle School, HS = High School, Col $=$ College, Pro $=$ Professional

differences were observed between sexes in the anterior reach direction or in composite score. While it may appear that there was not a difference between sexes in composite score, it is important to note that there was large variability in each sex, sport, and age/competition level in YBT-LQ performance. This was confirmed by the high heterogeneity observed indicating that there indeed may be differences in performance on the YBT-LQ among populations (i.e., age, sex, sport, activity, occupation, and injury status). This overall heterogeneity helped confirm that sex, sport, and competition level differences may exist. Thus, when the pooled means were analyzed, no differences were noted. Composite reach scores varied by as much as 13 \%LL de- pending on the sex, sport, and competition level. These differences may point to the differences seen in injury rate and type by sex. ${ }^{76}$

\section{SPORT DIFFERENCES}

There were significant differences observed between baseball and basketball in the posteromedial, posterolateral reach directions, and overall composite reach, with baseball demonstrating greater reach distances normalized to limb length. There were also differences observed between soccer and basketball in the anterior, posteromedial, posterolateral reach directions, and overall composite reach, with 


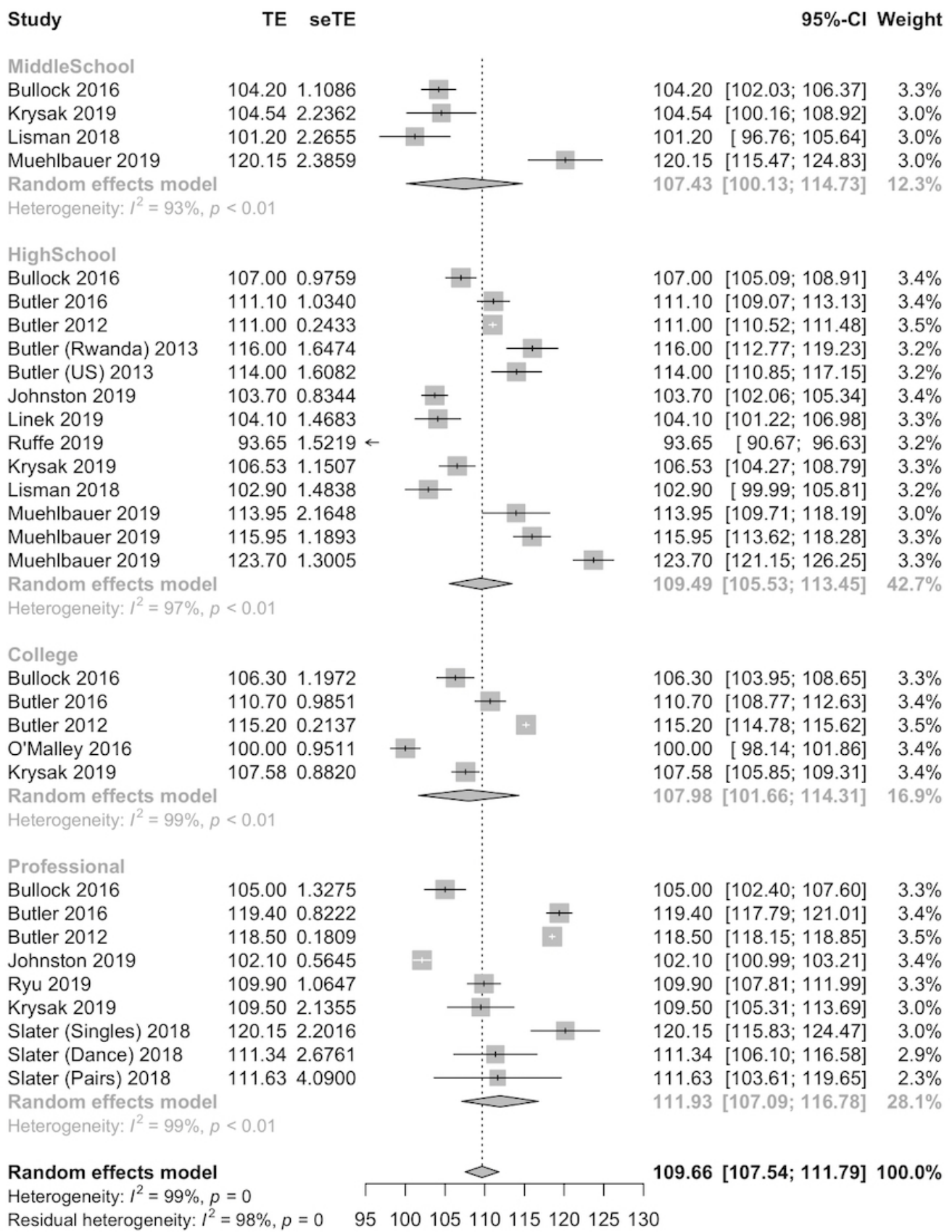

Figure 5: Pooled Y-Balance Test, Posteromedial Reach, Compared by Sport. MS = Middle School, HS = High School, Col $=$ College, Pro $=$ Professional

soccer demonstrating greater reach distances normalized to limb length. This may be due to sport specific adaptations in dynamic balance based on the demands and environment of the sport. For example, while both sports spend time running, soccer spends more time in unilateral stance at the limit of stability (e.g., kicking the ball) compared to basketball. ${ }^{77}$ While these differences may be due to sport specific adaptations, or limb dominance, specifically greater dynamic balance strategies on the stance leg during the kicking motion, it is also worth noting that dynamic neuromuscular control differences could be due to disparate anthropometric body types in athletes. For example, basketball players may in general have longer femurs than soccer players, which may make single limb squatting (i.e., anterior reach) biomechanically more difficult for basketball players.

\section{POPULATION DIFFERENCES SUMMARY}

There were significant differences across populations by sex and sport in YBT-LQ reach distance. There were not enough studies to analyze all the possible sex, sport, competition level permutations; however, it was clear that differences exist. For example, when male Rwandan high school soccer players were compared to male high school soccer players from the United States, the posteromedial and posterolat- 


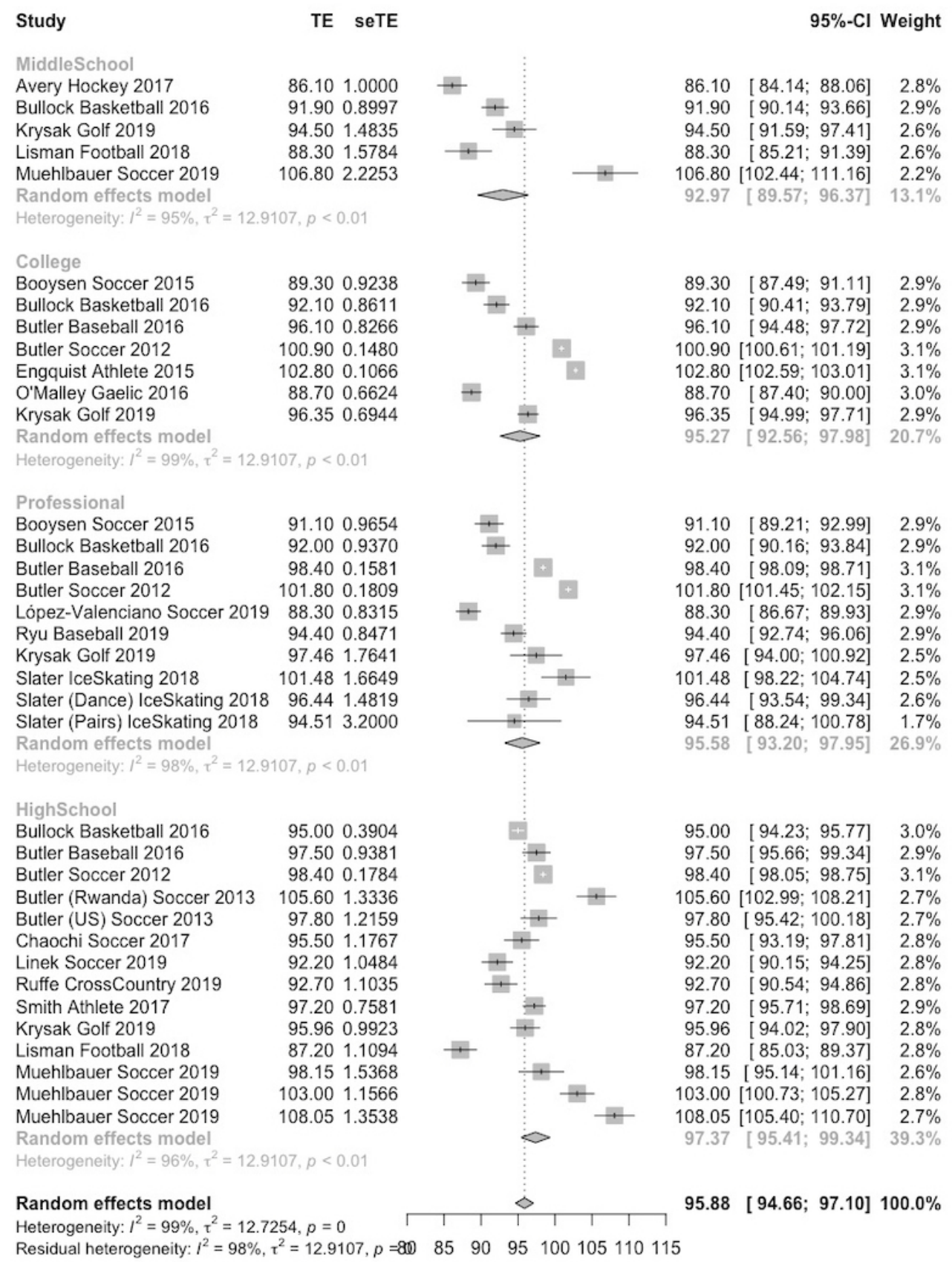

\section{Figure 6: Pooled Y-Balance Test, Posterolateral Reach, Compared by Sport. MS = Middle School, HS = High School, Col $=$ College, Pro $=$ Professional}

eral reach distances were not different. ${ }^{12}$ However, there was a significant difference in anterior reach and composite score. This shows YBT-LQ performance can potentially be affected by environment factors (e.g., in Rwanda there is less frequent wearing of athletic shoes and more frequent deep squatting for activities of daily living compared to the United States). ${ }^{12}$

It is interesting to note, that not only sex, sport, and environment might influence YBT-LQ performance, but also biological maturation. Researchers have found that YBT-LQ reach distance was significantly associated with the total Balance Error Scoring System score as YBT-LQ anterior and posteromedial reach distances. ${ }^{78}$

\section{INJURY PREDICTION VALIDITY OF THE YBT-LQ}

Since there were sport and gender differences in YBT-LQ, predictive studies could only be analyzed if they used a population specific cut point or examined homogeneous populations (e.g., male collegiate football players). Cut points for asymmetry and composite score varied

between studies. Due to these differences, composite score was found to be predictive of future injury in one study. ${ }^{3}$ More research is needed to develop these population-specific cut points to more accurately determine future injury risk.

Lehr et al. ${ }^{5}$ used population specific cut points across 
multiple sports. The researchers found that accurate injury risk identification was possible when multiple risk factors, including the YBT-LQ, were combined. The authors used age, sex, and sport specific risk cut points to place athletes in risk categories. These cut points were based on previously published injury prediction studies and normative databases. ${ }^{5}$ Thus, it is important to include age, sex, and sport cut points for injury risk identification. This study was not included in the meta-analysis since the researchers included multiple risk factors and the YBT-LQ was not able to be isolated as a risk factor. Further, Teyhen et al. ${ }^{79}$ found using a multifactorial model in soldiers that included YBTLQ: Anterior Reach $\leqslant 72 \%$ limb length as one of the risk factors in the model. This study further illustrates the point that YBT cut points are population specific but also that the YBT should be used as part of a multifactorial model rather than a single risk factor in isolation.

Six studies $34,36,39,48,50,68$ examined reach asymmetry as a predictor of injury. Four of the studies found a positive relationship between injury risk and reach asymmetry. However, there was variability in the definition of "asymmetry" with a wide cut point range and different risk reporting methods (e.g., odds ratios, likelihood ratio, sensitivity, and specificity). Thus, there may be an association with reach asymmetry and injury risk, but this was difficult to quantify given the variability of data reporting and analysis. Given that sport and sex differences were observed, it is likely that tolerance for asymmetry and direction of asymmetry may differ by sport or population. While asymmetry is an absolute value that is relative to the individual, it also may need population specific cut points, like composite score. A meta-analysis was not performed and definitive conclusions could not be drawn.

\section{LIMITATIONS}

While 57 articles were included in this review, there were not enough studies (even when combined) to provide enough power to compare populations by the different combinations of sex, sport, and competition levels. A metaanalysis on the YBT-LQ predictive ability was not completed because only two studies were found that used homogeneous methodology and reporting measures. YBTLQ reach asymmetry as a predictive factor was not analyzed due to the highly variable reported risk cut points. Two studies 9,40 were low quality, while the rest were moderate and high quality. Furthermore, some of the studies had high heterogeneity in the specific YBT-LQ methodology (hands free versus hands on hip, maximum versus average reach, etc.). Due to the study risk of bias stratification, and the methodological heterogeneity, these findings need to be taken with some caution. The YBT-LQ is a controlled dynamic balance test. As many sport injuries are sustained at high velocities and forces, the YBT-LQ does not mimic some sport mechanisms of injury, which decreases the transferability of these results to the sport setting. Finally, this systematic review investigated athletic and active populations; thus, these findings cannot be generalized to all adult populations (inactive adults, geriatrics, etc.).

\section{RECOMMENDATIONS FOR FUTURE RESEARCH}

From this meta-analysis, it is clear that populations when stratified by sex and sport perform significantly differently on the YBT-LQ. This has two large implications. First, future research needs to establish normative data for a wide range of populations that utilize this test. Second, injury predictive studies need to use population specific (e.g., age, sex, sport/activity) cut points for composite score and reach asymmetry. For asymmetry, these cut points should be greater than the standard error of measure $(3.2 \mathrm{~cm}),{ }^{9}$ so that meaningful asymmetry, beyond the error of measure, can be identified. Further, given the findings of Lehr et al. ${ }^{5}$ and Teyhen et al. ${ }^{79}$ it may be most appropriate to combine the YBT-LQ asymmetry and composite score specific to age, sex, and sport, along with other testing to accurately determine injury risk. Interestingly, country of origin seemed to impact performance; thus, cut points may need to specify beyond the aforementioned factors to include geographical location. Future research should use adequately powered and homogenous age, sex, and sport/activity specific analysis to determine if composite score is related to injury risk.

\section{CONCLUSION}

The YBT-LQ is a reliable tool for capturing dynamic single leg neuromuscular control at the limits of stability. Performance on the YBT-LQ differs based on age, sex, and sport, therefore clinicians should consider these factors when interpreting results to ensure accurate clinical decision-making. The relationship between the YBT-LQ and future injury risk remains unclear; future studies should utilize population specific cut points and homogenous samples to determine utility in injury prediction.

\section{DATA SHARING STATEMENT}

This study is registered with PROSPERO, and the protocol can be found at https://www.crd.york.ac.uk/prospero/ with the identifier Prospero CRD42018090102.

\section{CONFLICTS OF INTEREST}

Funding for payment of a graduate research assistant was made possible through the Ridgeway 488 Student Research Award from the University of Evansville.

Dr Phillip Plisky developed the Y-Balance Test Protocol and Test kit and receives royalties from the sale of the YBalance Test kit.

Submitted: December 09, 2020 CDT, Accepted: June 15, 2021

CDT 
This is an open-access article distributed under the terms of the Creative Commons Attribution 4.0 International License (CCBY-NC-SA-4.0). View this license's legal deed at https://creativecommons.org/licenses/by-nc-sa/4.0 and legal code at https://creativecommons.org/licenses/by-nc-sa/4.0/legalcode for more information. 


\section{REFERENCES}

1. Darrow CJ, Collins CL, Yard EE, Comstock RD. Epidemiology of severe injuries among United States high school athletes: 2005-2007. Am J Sports Med. 2009;37(9):1798-1805. doi:10.1177/036354650933301 $\underline{5}$

2. Aman M, Forssblad M, Henriksson-Larsén K. Insurance claims data: A possible solution for a national sports injury surveillance system? An evaluation of data information against ASIDD and consensus statements on sports injury surveillance. BMJ Open. 2014;4(6):e005056. doi:10.1136/bmjope n-2014-005056

3. Butler RJ, Lehr ME, Fink ML, Kiesel KB, Plisky PJ. Dynamic balance performance and noncontact lower extremity injury in college football players: An initial study. Sports Health. 2013;5(5):417-422. doi:10.1177/1 $\underline{941738113498703}$

4. Plisky PJ, Rauh MJ, Kaminski TW, Underwood FB. Star Excursion Balance Test as a predictor of lower extremity injury in high school basketball players. $J$ Orthop Sports Phys Ther. 2006;36(12):911-919. doi:1 $\underline{0.2519 / j o s p t .2006 .2244}$

5. Lehr ME, Plisky PJ, Butler RJ, Fink ML, Kiesel KB, Underwood FB. Field-expedient screening and injury risk algorithm categories as predictors of noncontact lower extremity injury. Scand J Med Sci Sports. 2013;23(4):225-232. doi:10.1111/sms.12062

6. Gribble PA, Hertel J, Plisky P. Using the Star Excursion Balance Test to assess dynamic posturalcontrol deficits and outcomes in lower extremity injury: A literature and systematic review. J Athl Train. 2012;47(3):339-357. doi:10.4085/1062-6050-4 7.3.08

7. Steib S, Zahn P, Zu Eulenburg C, Pfeifer K, Zech A. Time-dependent postural control adaptations following a neuromuscular warm-up in female handball players: A randomized controlled trial. BMC Sports Sci Med Rehabil. 2016;8:33. doi:10.1186/s1310 2-016-0058-5

8. Steffen K, Emery CA, Romiti M, et al. High adherence to a neuromuscular injury prevention programme (FIFA 11+) improves functional balance and reduces injury risk in Canadian youth female football players: A cluster randomised trial. $\mathrm{Br} \mathrm{J}$ Sports Med. 2013;47(12):794-802. doi:10.1136/bjsport s-2012-091886
9. Plisky PJ, Gorman PP, Butler RJ, et al. The reliability of an instrumented device for measuring components of the star excursion balance test. $\mathrm{N} \mathrm{Am} \mathrm{J}$ Sports Phys Ther. 2009;4:92-99. http://www.ncbi.nl m.nih.gov/pubmed/21509114.

10. Coughlan GF, Fullam K, Delahunt E, Gissane C, Caulfield BM, Sci M. A Comparison Between Performance on Selected Directions of the Star Excursion Balance Test and the Y Balance Test. J Athl Train. 2012;47(4):366-371. doi:10.4085/1062-6050-4 7.4.03

11. Fullam K, Caulfield B, Coughlan GF, Delahunt E. Kinematic Analysis of Selected Reach Directions of the Star Excursion Balance Test Compared with the YBalance Test. J Sport Rehabil. 2014;23(1):27-35. doi:1 0.1123/jsr.2012-0114

12. Butler RJ, Queen RM, Beckman B, et al. Comparison of dynamic balance in adolescent male soccer players from rwanda and the United States. Int J Sports Phys Ther. 2013;8:749-755. https://www.ncb i.nlm.nih.gov/pubmed/24377061.

13. Butler RJ, Southers C, Gorman PP, Kiesel KB, Plisky PJ. Differences in soccer players' dynamic balance across levels of competition. J Athl Train. 2012;47(6):616-620. doi:10.4085/1062-6050-47.5.14

14. Butler RJ, Bullock G, Arnold T, Plisky P, Queen R. Competition-Level Differences on the Lower Quarter Y-Balance Test in Baseball Players. J Athl Train. 2016;51(12):997-1002. doi:10.4085/1062-6050-51.1 $\underline{2.09}$

15. Moher D, Shamseer L, Clarke M, et al. Preferred reporting items for systematic review and metaanalysis protocols (PRISMA-P) 2015 statement. Syst Rev. 2015;4:1. doi:10.1186/2046-4053-4-1

16. Phillips B, Ball C, Badenoch D, et al. Oxford centre for evidence-based medicine levels of evidence. $B J U$ Int. 2001;107:870. https://insights.ovid.com/bju-inter national/bjui/2011/03/000/oxford-centre-evidence-ba sed-medicine-levels-may/39/00125504.

17. Downs SH, Black N. The feasibility of creating a checklist for the assessment of the methodological quality both of randomised and non-randomised studies of health care interventions. J Epidemiol Community Health. 1998;52(6):377-384. doi:10.1136/j ech.52.6.377 
18. Mosler AB, Agricola R, Weir A, Hölmich P, Crossley KM. Which factors differentiate athletes with hip/groin pain from those without? A systematic review with meta-analysis. Br J Sports Med.

2015;49(12):810. doi:10.1136/bjsports-2015-094602

19. Wells G. The Newcastle-Ottawa Scale (NOS) for assessing the quality of nonrandomised studies in meta-analysis. 2004. https://ci.nii.ac.jp/naid/1002059 $\underline{0649 / .}$.

20. DerSimonian R, Laird N. Meta-analysis in clinical trials. Control Clin Trials. 1986;7(3):177-188. doi:10.1 $\underline{016 / 0197-2456(86) 90046-2}$

21. Borenstein M, Hedges LV, Higgins JPT, et al. Introduction to Meta-Analysis. John Wiley \& Sons; 2011. https://market.android.com/details?id=book-JO g9jdrq26wC.

22. Schwarzer G. meta: An R package for metaanalysis. $R$ News. 2007;7:40-45. https://cran.r-projec t.org/doc/Rnews/Rnews_2007-3.pdf.

23. Alnahdi AH, Alderaa AA, Aldali AZ, Alsobayel H. Reference values for the $Y$ Balance Test and the lower extremity functional scale in young healthy adults. $J$ Phys Therapy Sci. 2015;27(12):3917-3921. doi:10.158 9/ipts.27.3917

24. Avery M, Wattie N, Holmes M, Dogra S. Seasonal Changes in Functional Fitness and Neurocognitive Assessments in Youth Ice-Hockey Players. J Strength Cond Res. 2018;32(11):3143-3152. doi:10.1519/jsc.000 $\underline{0000000002399}$

25. Benis R, Bonato M, La Torre AL. Elite Female Basketball Players' Body-Weight Neuromuscular Training and Performance on the Y-Balance Test. J Athl Train. 2016;51(9):688-695. doi:10.4085/1062-605 $\underline{0-51.12 .03}$

26. Booysen MJ, Gradidge PJ-L, Watson E. The relationships of eccentric strength and power with dynamic balance in male footballers. J Sports Sci. 2015;33(20):2157-2165. doi:10.1080/02640414.2015.1 $\underline{064152}$

27. Bullock GS, Arnold TW, Plisky PJ, Butler RJ. Basketball Players' Dynamic Performance Across Competition Levels. J Strength Cond Res. 2018;32(12):3528-3533. doi:10.1519/jsc.00000000000 $\underline{01372}$

28. Chaouachi M, Granacher U, Makhlouf I, et al. Within Session Sequence of Balance and Plyometric Exercises Does Not Affect Training Adaptations with Youth Soccer Athletes. J Sports Sci Med. 2017;16:125-136. https://www.ncbi.nlm.nih.gov/pub med/28344461.
29. Chimera NJ, Kremer K. SportsmetricsTM training improves power and landing in high school rowers. Int J Sports Phys Ther. 2016;11:44-53. https://www.nc bi.nlm.nih.gov/pubmed/26900499.

30. Chimera NJ, Smith CA, Warren M. Injury history, sex, and performance on the functional movement screen and Y balance test. J Athl Train. 2015;50:475-485. doi:10.4085/1062-6050-49.6.02

31. Cosio-Lima L, Knapik JJ, Shumway R, et al. Associations between functional movement screening, the Y balance test, and injuries in coast guard training. Mil Med. 2016;181(7):643-648. doi:1 $\underline{0.7205 / \text { milmed-d-15-00208 }}$

32. Engquist KD, Smith CA, Chimera NJ, Warren M. Performance comparison of student-athletes and general college students on the functional movement screen and the Y balance test. J Strength Cond Res. 2015;29(8):2296-2303. doi:10.1519/jsc.000000000000 $\underline{0906}$

33. Faigenbaum AD, Myer GD, Fernandez IP, et al. Feasibility and reliability of dynamic postural control measures in children in first through fifth grades. Int $J$ Sports Phys Ther. 2014;9:140-148. https://www.ncbi.n lm.nih.gov/pubmed/24790775.

34. Gonell AC, Romero JAP, Soler LM. Relationship between the Y balance test scores and soft tissue injury incidence in a soccer team. Int J Sports Phys Ther. 2015;10(955). https://www.ncbi.nlm.nih.gov/pm c/articles/pmc4675196/.

35. Gorman PP, Butler RJ, Rauh MJ, et al. Differences in dynamic balance scores in one sport versus multiple sport high school athletes. Int J Sports Phys Ther. 2012;7:148-153. https://www.ncbi.nlm.nih.gov/ pubmed/22530189.

36. Hartley EM, Hoch MC, Boling MC. Y-balance test performance and BMI are associated with ankle sprain injury in collegiate male athletes. J Sci Med Sport. 2018;21(7):676-680. doi:10.1016/j.jsams.2017.1 $\underline{0.014}$

37. Hoch MC, Welsch LA, Hartley EM, Powden CJ, Hoch JM. Y-Balance Test Performance After a Competitive Field Hockey Season: A Pretest-Posttest Study. J Sport Rehabil. 2017;26(5). doi:10.1123/jsr.201 7-0004

38. Hudson C, Garrison JC, Pollard K. Y-balance normative data for female collegiate volleyball players. Physical Therapy in Sport. 2016;22:61-65. do i:10.1016/i.ptsp.2016.05.009 
39. Lai WC, Wang D, Chen JB, Vail J, Rugg CM, Hame SL. Lower quarter Y-balance test scores and lower extremity injury in NCAA Division I athletes. Orthop J Sports Med. 2017;5(8):232596711772366. doi:10.1177/ 2325967117723666

40. Linek P, Sikora D, Wolny T, Saulicz E. Reliability and number of trials of Y Balance Test in adolescent athletes. Musculoskeletal Science and Practice. 2017;31:72-75. doi:10.1016/j.msksp.2017.03.011

41. Miller MM, Trapp JL, Post EG, et al. The effects of specialization and sex on anterior Y-balance performance in high school athletes. Sports Health. 2017;9(4):375-382. doi:10.1177/1941738117703400

42. de la Motte SJ, Lisman P, Sabatino M, Beutler AI, O'Connor FG, Deuster PA. The relationship between functional movement, balance deficits, and previous injury history in deploying marine warfighters. $J$ Strength Cond Res. 2016;30(6):1619-1625. doi:10.151 9/jsc.0000000000000850

43. de la Motte SI, Gribbin TC, Lisman P, Beutler AI, Deuster P. The interrelationship of common clinical movement screens: Establishing population-specific norms in a large cohort of military applicants. J Athl Train. 2016;51(11):897-904. doi:10.4085/1062-6050-5 1.9.11

44. O’Malley E, Murphy JC, McCarthy Persson U, Gissane C, Blake C. The effects of the Gaelic athletic Association 15 training program on neuromuscular outcomes in Gaelic football and hurling players: A randomized cluster trial. J Strength Cond Res. 2017;31(8):2119-2130. doi:10.1519/jsc.000000000000 $\underline{1564}$

45. Rössler R, Donath L, Bizzini M, Faude O. A new injury prevention programme for children's football FIFA 11+ Kids - can improve motor performance: A cluster-randomised controlled trial. J Sports Sci. 2016;34(6):549-556. doi:10.1080/02640414.2015.1099 $\underline{715}$

46. Shaffer SW, Teyhen DS, Lorenson CL, et al. Ybalance test: A reliability study involving multiple raters. Military Medicine. 2013;178(11):1264-1270. do $\mathrm{i}: 10.7205 /$ milmed-d-13-00222

47. Schlingermann BE, Lodge CA, Gissane C, Rankin PM. Effects of the Gaelic athletic association 15 on lower extremity injury incidence and neuromuscular functional outcomes in collegiate gaelic games. $J$ Strength Cond Res. 2018;32(7):1993-2001. doi:10.151 9/jsc.0000000000002108

48. Teyhen DS, Shaffer SW, Butler RJ, et al. What risk factors are associated with musculoskeletal injury in US army rangers? A prospective prognostic study. Clin Orthop Relat Res. 2015;473(9):2948-2958. doi:10.100 7/s11999-015-4342-6
49. Teyhen DS, Rhon DI, Butler RJ, et al. Association of physical inactivity, weight, smoking, and prior injury on physical performance in a military setting. $J$ Athl Train. 2016;51(11):866-875. doi:10.4085/1062-60 50-51.6.02

50. Wright AA, Dischiavi SL, Smoliga JM, Taylor JB, Hegedus EJ. Association of lower quarter Y-balance test with lower extremity injury in NCAA Division 1 athletes: An independent validation study.

Physiotherapy. 2017;103(2):231-236. doi:10.1016/i.ph ysio.2016.06.002

51. Greenberg ET, Barle M, Glassmann E, Jung M-K. Interrater and test-retest reliability of the Y Balance Test in healthy, early adolescent female athletes. Intl J Sports Phys Ther. 2019;14(2):204-213. doi:10.26603/ij spt20190204

52. Kenny SJ, Palacios-Derflingher L, Owoeye OBA, Whittaker JL, Emery CA. Between-day reliability of pre-participation screening components in preprofessional ballet and contemporary dancers. $J$ Dance Med Sci. 2018;22(1):54-62. doi:10.12678/108 9-313x.22.1.54

53. Lacey M, Donne B. Does fatigue impact static and dynamic balance variables in athletes with a previous ankle injury? Int J Exerc Sci. 2019;12(3):1121-1137.

54. Smith LJ, Creps JR, Bean R, Rodda B, Alsalaheen $B$. Performance and reliability of the Y-Balance Test $^{\mathrm{TM}}$ in high school athletes. J Sports Med Phys Fitness. 2018;58(11):1671-1675. doi:10.23736/S002 2-4707.17.07218-8

55. Teyhen DS, Riebel MA, McArthur DR, et al. Normative data and the influence of age and gender on power, balance, flexibility, and functional movement in healthy service members. Mil Med. 2014;179(4):413-420. doi:10.7205/milmed-d-13-0036 $\underline{2}$

56. Bonato M, Benis R, La Torre A. Neuromuscular training reduces lower limb injuries in elite female basketball players. A cluster randomized controlled trial. Scand J Med Sci Sports. 2018;28(4):1451-1460. o i:10.1111/sms.13034

57. Brumitt J, Nelson K, Duey D, Jeppson M, Hammer L. Preseason Y Balance Test scores are not associated with noncontact time-loss lower quadrant injury in male collegiate basketball players. Sports. 2018;7(1):4. doi:10.3390/sports7010004

58. Johnston W, Duignan C, Coughlan GF, Caulfield B. Dynamic balance performance varies by position but not by age group in elite Rugby Union players - a normative study. J Sports Sci. 2019;37(11):1308-1313. doi:10.1080/02640414.2018.1557360 
59. Krysak S, Harnish CR, Plisky PJ, Knab AM, Bullock GS. Fundamental movement and dynamic balance disparities among varying skill levels in golfers. Int $J$ Sports Phys Ther. 2019;14(4):537-545. doi:10.26603/ijs pt20190537

60. Linek P, Booysen N, Sikora D, Stokes M. Functional movement screen and Y balance tests in adolescent footballers with hip/groin symptoms. Phys Ther Sport. 2019;39:99-106. doi:10.1016/i.ptsp.2019.0 $\underline{7.002}$

61. Lisman P, Nadelen M, Hildebrand E, Leppert K, de la Motte S. Functional movement screen and YBalance test scores across levels of American football players. Biol Sport. 2018;35(3):253-260. doi:10.5114/b iolsport.2018.77825

62. López-Valenciano A, Ayala F, De Ste Croix M, Barbado D, Vera-Garcia FJ. Different neuromuscular parameters influence dynamic balance in male and female football players. Knee Surg Sports Traumatol Arthrosc. 2019;27(3):962-970. doi:10.1007/s00167-01 8-5088-y

63. Muehlbauer T, Schwiertz G, Brueckner D, Kiss R, Panzer S. Limb differences in unipedal balance performance in young male soccer players with different ages. Sports. 2019;7(1):20. doi:10.3390/sport $\underline{\text { s7010020 }}$

64. Ruffe NJ, Sorce SR, Rosenthal MD, Rauh MJ. Lower quarter- and upper quarter Y Balance Tests as predictors of running-related injuries in high school cross-country runners. Int J Sports Phys Ther. 2019;14(5):695-706. doi:10.26603/ijspt20190695

65. Ryu CH, Park J, Kang M, et al. Differences in lower quarter Y-balance test with player position and ankle injuries in professional baseball players. J Orthop Surg. 2019;27(1):1-7. doi:10.1177/2309499019832421

66. Slater LV, Vriner M, Schuyten K, Zapalo P, Hart JM. Sex differences in Y-Balance performance in elite figure skaters. J Strength Cond Res. 2020;34(5):1416-1421. doi:10.1519/jsc.000000000000 $\underline{2542}$

67. Smith J, DePhillipo N, Azizi S, et al. The Lower Extremity Grading System (LEGS) to evaluate baseline lower extremity performance in high school athletes. Int J Sports Phys Ther. 2018;13(3):401-409. d oi:10.26603/ijspt20180401

68. Smith CA, Chimera NJ, Warren M. Association of y balance test reach asymmetry and injury in division I athletes. Med Sci Sports Exerc. 2015;47(1):136-141. do i: $10.1249 / \mathrm{mss} .0000000000000380$
69. de la Motte SJ, Clifton DR, Gribbin TC, Beutler AI, Deuster PA. Functional movement assessments are not associated with risk of injury during military basic training. Mil Med. 2019;184(11-12):e773-e780. doi:1 0.1093/milmed/usz118

70. Gonzalez SL, Diaz AM, Plummer HA, Michener LA. Musculoskeletal screening to identify female collegiate rowers at risk for low back pain. J Athl Train. 2018;53(12):1173-1180. doi:10.4085/1062-605 $\underline{0-50-17}$

71. Johnston W, O’Reilly M, Duignan C, et al. Association of dynamic balance with sports-related concussion: A prospective cohort study. Am J Sports Med. 2019;47(1):197-205. doi:10.1177/036354651881 $\underline{2820}$

72. Lisman P, Hildebrand E, Nadelen M, Leppert K. Association of functional movement screen and Ybalance test scores with injury in high school athletes. J Strength Cond Res. 2019;10.1519/ ISC. 0000000000003082 . doi:10.1519/jsc.00000000000 $\underline{03082}$

73. Šiupšinskas L, Garbenytė-Apolinskienè T, Salatkaitė S, Gudas R, Trumpickas V. Association of pre-season musculoskeletal screening and functional testing with sports injuries in elite female basketball players. Sci Rep. 2019;9(1). doi:10.1038/s41598-019-4 $\underline{5773-0}$

74. Vaulerin J, Chorin F, Emile M, d'ArripeLongueville F, Colson SS. Ankle sprains risk factors in a sample of French firefighters: A preliminary prospective study. J Sport Rehabil. 2020;29(5):1-23. do $\mathrm{i}: 10.1123 /$ jsr.2018-0284

75. Steindl R, Kunz K, Schrott-Fischer A, Scholtz A. Effect of age and sex on maturation of sensory systems and balance control. Dev Med Child Neurol. 2006;48(6):477-482. doi:10.1017/s0012162206001022

76. Powell JW, Barber-Foss KD. Sex-related injury patterns among selected high school sports. Am J Sports Med. 2000;28(3):385-391. doi:10.1177/0363546 $\underline{5000280031801}$

77. Cowley HR, Ford KR, Myer GD, et al. Differences in neuromuscular strategies between landing and cutting tasks in female basketball and soccer athletes. J Athl Train. 2006;41:67-73. https://www.ncbi.nlm.ni h.gov/pubmed/16619097.

78. John C, Rahlf AL, Hamacher D, Zech A. Influence of biological maturity on static and dynamic postural control among male youth soccer players. Gait \& Posture. 2019;68:18-22. doi:10.1016/j.gaitpost.2018.1 $\underline{0.036}$ 
79. Teyhen DS, Shaffer SW, Goffar SL, et al.

Identification of risk factors prospectively associated

with musculoskeletal injury in a warrior athlete

population. Sports Health. 2020;12(6):564-572. doi:1

$\underline{0.1177 / 1941738120902991}$ 